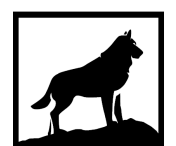

Michigan Technological

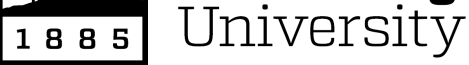

Michigan Technological University Digital Commons @ Michigan Tech

\title{
LIGHT PROPAGATION THROUGH A TURBULENT CLOUD: COMPARISON OF MEASURED AND COMPUTED EXTINCTION
}

Eduardo Rodriguez-feo Bermudez

Michigan Technological University, eduardor@mtu.edu

Copyright 2019 Eduardo Rodriguez-feo Bermudez

\section{Recommended Citation}

Rodriguez-feo Bermudez, Eduardo, "LIGHT PROPAGATION THROUGH A TURBULENT CLOUD: COMPARISON OF MEASURED AND COMPUTED EXTINCTION", Open Access Master's Thesis, Michigan Technological University, 2019.

https://doi.org/10.37099/mtu.dc.etdr/909

Follow this and additional works at: https://digitalcommons.mtu.edu/etdr

Part of the Atmospheric Sciences Commons, Fluid Dynamics Commons, and the Optics Commons 
LIGHT PROPAGATION THROUGH A TURBULENT CLOUD: COMPARISON

OF MEASURED AND COMPUTED EXTINCTION

By

Eduardo Rodriguez-feo Bermudez

\begin{abstract}
A THESIS
Submitted in partial fulfillment of the requirements for the degree of MASTER OF SCIENCE

In Applied Physics

MICHIGAN TECHNOLOGICAL UNIVERSITY
\end{abstract}

2019

(C) 2019 Eduardo Rodriguez-feo Bermudez 

This thesis has been approved in partial fulfillment of the requirements for the Degree of MASTER OF SCIENCE in Applied Physics.

Department of Physics

Thesis Co-advisor: Dr. Raymond Shaw

Thesis Co-advisor: Dr. Claudio Mazzoleni

Committee Member: Dr. Will Cantrell

Department Chair: Dr. Ravindra Pandey 



\section{Dedication}

This report is dedicated to my friends and family who have stood beside me in times of scarcity and plenty. To those who have helped me witness both the sunny weather of South Florida, and the starry night of Northern Michigan. Our interactions have molded me into the person I am today, and for that I will be eternally grateful. 



\section{Contents}

List of Figures $\ldots \ldots \ldots \ldots \ldots \ldots \ldots \ldots \ldots$

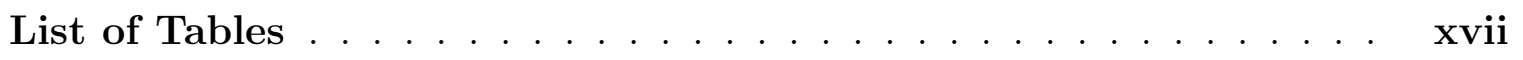

Acknowledgments ....................

List of Abbreviations ..................

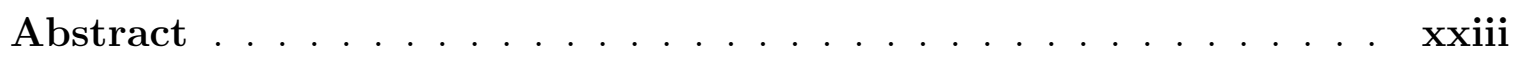

1 Introduction ........................... 1

1.1 Introduction . . . . . . . . . . . . . . . . . 1

1.1.1 Proposed Question ................ 1

1.1 .2 Importance . . . . . . . . . . . . . . . . 2

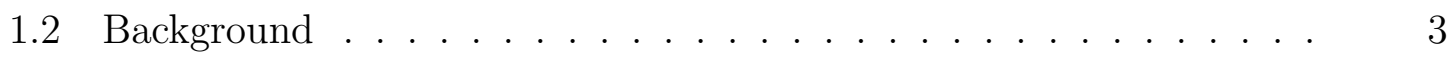

1.2.1 Theoretical Background $\ldots \ldots \ldots \ldots \ldots$

1.2.2 Computational/Experimental Background ........ 5

1.3 Experiment and Equipment $\ldots \ldots \ldots \ldots \ldots$

$1.3 .1 \quad$ Experiment $\ldots \ldots \ldots \ldots \ldots \ldots \ldots$ 
1.3 .2 Schematic .................... 9

1.3.3 $\pi$-Chamber ..................... 9

1.3.4 Laser and Photo-Diodes ............. 12

1.3.5 Data Acquisition ................. 14

1.3.6 WELAS ...................... 14

2 Procedures \& Experiment . . . . . . . . . . . . . . . . 17

2.1 Procedures for Relative Humidity Readings . . . . . . . . . . . . . . 17

2.1.1 Optical Depth Readings, Laser-Hygrometer . . . . . . . 21

2.1.2 Optical Depth Readings, WELAS . . . . . . . . . 22

2.1.3 Optical Depth Comparison . . . . . . . . . . 26

3 Results and Discussion . . . . . . . . . . . . . . . . 29

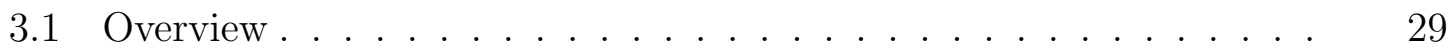

3.2 Relative Humidity Results . . . . . . . . . . . . . . . . . . . 30

3.3 WELAS and Mie Scattering Optical Depth . . . . . . . . . 31

3.3.1 Results........................... 31

3.3.2 Analysis ..................... 33

3.4 Laser - Hygrometer Optical Depth . . . . . . . . . . . . . 36

3.4 .1 Results...................... 36

3.4 .2 Analysis ..................... 36

3.5 Hygrometer and WELAS Comparison . . . . . . . . . . 38

3.5.1 Results....................... 38 


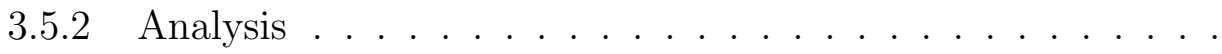

4 Conclusion ............................. 41

4.1 Overall Implications . . . . . . . . . . . . . . . 41

4.2 Limitations and Issues . . . . . . . . . . . . . . . 42

4.3 Future Research Possibilities . . . . . . . . . . . . . . . . . . 44

References ............................... 47

A Other Plots and Images . . . . . . . . . . . . . . . . 53

B Letters of Permission . . . . . . . . . . . . . . . . 55

B.1 Permission From AMS . . . . . . . . . . . . . . 55

B.2 Elsevier ......................... 56 



\section{List of Figures}

1.1 Relative intensity of light as a function of propagation distance. Where the solid line is from a uniform medium and in agreement with BeerLambert. Top line is for positively correlated non-uniform medium, and lower line is for negatively correlated non-uniform medium Shaw et al. 2002). Permission was granted by JQSRT for use this figure.

6

1.2 An example of light scattering in a Monte Carlo simulation. Image provided by Corey Packard. Note that most of the particles are scattered in the forward direction with a very small deviation from the

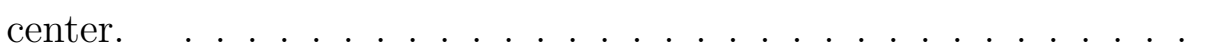

1.3 A schematic representation of the experimental setup for the LaserHygrometer. Photo-Diode ai1 is the reference, and Photo-Diode ai0 is the experimental $\ldots \ldots \ldots \ldots \ldots \ldots$

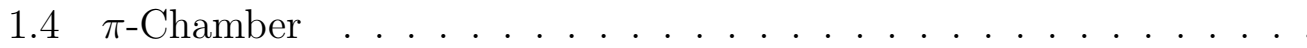

(a) Schematic view of the $\pi$-Chamber from Chandrakar et al., 2016).

Permission was granted by AMS to use this figure. . . . . . 
(b) Cross-Section of a turbulent Cloud Inside the Chamber, Image provided by Kamal Kant Chandrakar . . . . . . . . . . .

11

1.5 Pictures of the optical set-up including the NIR Laser and Photodiodes. The laser beam passes through to outside windows, NBK-7 2 "diameter and $12 \mathrm{~mm}$ thickness windows with the $\mathrm{C}$ coating. And two inner windows, made up of float glass of 3/16 thickness. All windows were chosen because of their low absorption and high transmission rates around $1392 \mathrm{~nm}$ wavelength range. . . . . . . . . . . . . 13

(a) Laser and Reference Photo-diode . . . . . . . . . . . . . 13

(b) Experimental Photo-diode ................ 13

2.1 An example of how data is acquired using the Laser-Hygrometer. The first plot shows the true readings from both photo-diodes as it scans the AoI by changin the $V_{\text {bias }}$. Second plot is the wavelength output of the laser at a given $V_{\text {bias }}$ for a $V_{\text {tec }}$ of $0.4 \mathrm{~V}$. The last plot represents the converted values of voltage at specific wave-numbers, centered about the point which we focus on the transmission rate . . . . . . 19

2.2 The above plot shows the water vapor absorption lines in the AoI. The $X S_{a b s}$ values of water at a given wave-number are extracted for further analysis into the $\mathrm{RH}$ inside the $\pi$-Chamber. The data that the plot consist of was retrieved from the HITRAN database. . . . . . . . . 
2.3 The scattering angle dependence of light as it comes into contact with different sized particles, at a refractive index of 1.33. Additionally, the forward scattering angle deficiency is shown for the case where the particle is located $\approx 2.2 \mathrm{~m}$ away from the experimental photo-diode. The plots were created using the code from Christian Matzler Matzler, 2002). The size parameters chosen nearly correspond to actual size parameters calculated from the DSD. In terms of droplet radius the polar plots correspond to (a) $\mathrm{R}=0.022 \mu m$,(b) $\mathrm{R}=0.22 \mu m$, (c) $\mathrm{R}=$ $2.2 \mu m,(\mathrm{~d}) \mathrm{R}=19.9 \mu \mathrm{m}$. Note that as the size parameter increases, the ratio of area within the forward scatting deficiency, relative to the area outside of it, increases. . . . . . . . . . . . . .

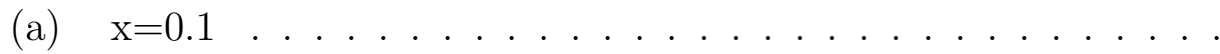

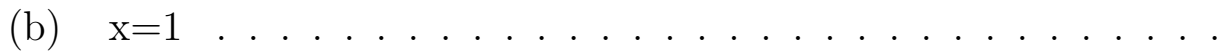

(c)

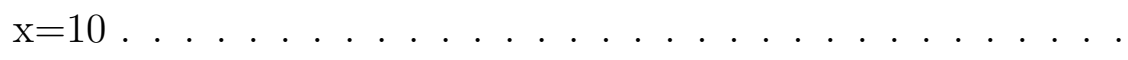

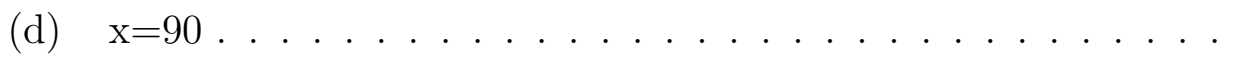

2.4 The forward scattering efficiency for three different distances with respect to the experimental photo-diode inside the $\pi$-Chamber, $2.2 \mathrm{~m}$, $1.2 \mathrm{~m}, 0.2 \mathrm{~m} . \ldots \ldots \ldots \ldots \ldots$ 
3.1 Relative humidity readings inside the $\pi$-Chamber, The chamber computer was reading an $R H \%$ of $88 \%$. The $3 \%$ discrepancy corresponds to an OD difference of 0.01 . This number was acquired by working backwards on equations (2.1)-(2.4). Blue line represent a poly-fitted line of the $10^{\text {th }}$ order degree. While the red-line is one standard deviation from it, both above and below. .............

3.2 The particle size distribution inside the $\pi$ - Chamber at three different time periods. The bi-modal nature of the distribution is due to the presence of activated droplets and non-activated hydrated particles. The charts include the calculated OD of the size distribution. The Mie scattering calculations above were done under the scenario where the mean particle location was in the middle of the $\pi$-Chamber, $1.2 \mathrm{~m}$ away from the experimental photo-diode. . . . . . . . . . .

(a) Reference background particle size distribution . . . . . . 32

(b) Particle size distribution at $\approx 10$ minutes . . . . . . 32

(c) Particle size distribution at $\approx 50$ minutes. . . . . . . . . 32 
3.3 Laser - Hygrometer results showing the OD before and after the introduction of aerosols into the cloud chamber. The extinction caused by the water vapor, represented by the first mean line, is subtracted from the overall extinction after 90 minutes, represented by the second mean line. This results in the measured OD only being due to light scattering from water droplets. The dashed green line represents a running mean algorithm of 3 index values, which corresponds to a total of 90 seconds. The red lines represent one standard deviation from the mean, both below and above. Resulting in a capture of $\approx 68 \%$ of all data points taken from the Laser-Hygrometer. . . . . . . . . . .

(a) Hygrometer Results at 5K/m Gradient . . . . . . . . 37

(b) Hygrometer Results at $10 \mathrm{~K} / \mathrm{m}$ Gradient . . . . . . . . .

(c) Hygrometer Results at 15K/m Gradient . . . . . . . . .

(d) Hygrometer Results at 20K/m Gradient . . . . . . . . . . 
3.4 The OD results of both the WELAS and the Laser-Hygrometer. Temperature difference here represents the temperature between the floor and ceiling inside the $\pi$-Chamber. The error margin for the WELAS comes from the uncertainty for the location of the particles inside the $\pi$-Chamber with respect to the experimental photo-diode. The errors for the Laser-Hygrometer come from the oscillating nature of the transmission ratio during the experiment. This sources of the oscillation could be from experimental equipment such as $V_{T e c}, V_{\text {Bias }}$ signal not outputting correctly, and/or rapid temperature changes causing fluctuations of the lasers emitted wavelength. Another possible source of the fluctuation could be due to the particle clustering due to turbulence inside the $\pi$-Chamber. They represent one standard deviations above and below the mean OD value, capturing up to $68 \%$ of possible

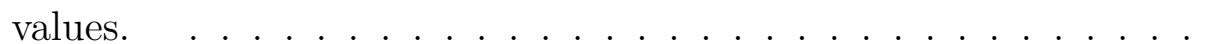

A.1 This is the result obtained when the windows become foggy. A maximum optical depth of $\approx 4$ is reached. When that occurs the experimental photo-diode is flattened out and the calculations of optical depth become infinity due to a division by zero. . . . . . . . . 54

(a) Laser-Hygrometer Data ................ 54

(b) Optical Depth Line . . . . . . . . . ..... 54 


\section{List of Tables}

3.1 Overall Optical Depth at Different Distances, and Asymmetric Param-

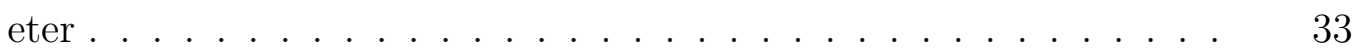

3.2 OD before, and after aerosol influx. Also included is their combine standard deviation to serve as an uncertainty factor. . . . . . . . 



\section{Acknowledgments}

I Would like to acknowledge the Cloud Chamber group, whose individuals have educated me when I found myself lost in the field of Applied Physics and Atmospheric Sciences, their aid allowed for the research to overcome road blocks that I may not have been able to by myself. So special thanks to my peers, Subin, Jesse, Kamal, Shawon, Neel, Elise, and Susan. Additionally I want to acknowledge those who joined us momentarily and left great memories along the way, Susanne, and Prasanth. And lastly I want to acknowledge Dr. Cantrell who played a key role in managing and guiding the entire group.

I would like to acknowledge Greg Kinney who made the research possible by providing ideas and feedback for research and experiments through the last two years. He also gave support when it came to setting up the experiment, as well as data taking. Additionally I would like to also Acknowledge Tyler Capek who taught me much about optics and laid the foundation to the Hygrometer Schematic.

And finally I would like to Acknowledge my advisors, Dr. Shaw, and Dr. Mazzoleni who guided me at every step of my graduate career without hesitation. 



\section{List of Abbreviations}

$\begin{array}{ll}\text { AoI } & \text { Area of Interest } \\ \text { AoA } & \text { Angle of Arrival } \\ \text { DSD } & \text { Droplet Size Distribution } \\ \text { HITRAN } & \text { High-Resolution Transmission of molecular absorption } \\ \text { LWC } & \text { Liquid Water Content } \\ \text { MATLAB } & \text { Matrix Laboratory } \\ \text { MTU } & \text { Michigan Technological University } \\ \text { Na } & \text { Avogadro's number, 6.0221409e+23 } \\ \text { NI-DAQmx } & \text { Data Acquisition Card from National Instruments } \\ \text { NIR } & \text { Near Infra Red } \\ \text { PDI } & \text { Phase Doppler Interferometer } \\ \text { PIV } & \text { Particle Image Velocimetry } \\ & \text { Relative Humidity Percentage } \\ & \end{array}$





\section{Abstract}

Remote sensing techniques used for measurement of atmospheric cloud properties operate under the notion that light extinction caused by scattering and absorption is exponential due to Beer-Lambert law. This is expected to be valid for a uniform medium with no spatial correlations between particle position. The aim of this research was to show that under turbulent conditions, cloud droplets cannot be interpreted as non-correlated, and in turn will exhibit a lower than exponential light decay from scattering. The research took place at the MTU $\pi$-Chamber laboratory. A temperature difference between the floor and ceiling of the chamber was applied to create convection-driven turbulence. When turbulent cloud conditions were achieved, it's optical depth properties was analyzed. This was done by deriving the optical depth by computational means through the acquisition of its droplet size distribution, and processing it through Mie scattering theory, while simultaneously acquiring direct measurement of optical depth using a Laser-Hygrometer. Results showed that there is a trend where larger temperature differences inside the chamber caused the direct extinction of light to deviate more strongly from the computed extinction. This less then exponential extinction parameter allows us to understand the significant effect that a turbulent cloud cover has on radar and satellite signals. 



\section{Chapter 1}

\section{Introduction}

\section{$1.1 \quad$ Introduction}

\subsubsection{Proposed Question}

Light propagation through turbulent media has been a topic of debate for many years. Previous research papers have talked about the existence of particle distributions whose extinction rates are lower than exponential and thus lower than what Beer-Lambert law rates would indicate (Kostinski, 2001). But this claim has been a topic of debate in the past $($ Borovoi, 2002$)$. Our research hopes to provide further insight into this topic by approaching it from an experimental methodology, 
rather than a theoretical or computational one. Our goal is to determine if there exists a non-trivial deviation for the rate of light extinction between what is to be expected from the Beer-Lambert law, and what the actual extinction is. This goal will be accomplished by undertaking two simultaneous analysis of a cloud under turbulent conditions inside the Michigan Technological University $\pi$-Chamber. The first analysis will be via computational/theoretical means where the Droplet Size Distribution(DSD) of the cloud will be taken and processed through Mie scattering theory code (Matzler, 2002). This will give us an extinction rate and optical depth (OD) that will be based under the assumption that the spatial distribution of particles in the medium are randomly homogeneous and non-correlated. The second analysis will be based on direct extraction of the light extinction inside the $\pi$-Chamber. A laser beam will propagate across the cloud and the difference between its initial and final intensity will be taken, and converted to a OD. Lastly we compare both analysis to see if we detect a non-trivial difference between their OD.

\subsubsection{Importance}

Optical properties of clouds have a direct effect on atmospheric, meteorological, and climate research. When remote sensing techniques consider the extinction of light caused by clouds, it is usually under the assumption that the OD of a cloud is uniform through the entirety of the path length. However, my research is to show that 
this established reasoning may need to be revised because of the in-homogeneity of turbulent clouds. A further understanding into light propagation within a turbulent medium may also give an explanation, and reduction to the uncertainties and inaccuracies sometimes found in atmospheric data from satellites (Stephens et al., 2018). Widening our understanding of this phenomena could lead to the establishment of a correction factor in remote sensing data analysis like that proposed in (Frankel et al. 2017). Where if the parameters of DSD and flow statistics are known, a correction factor can be created and applied for exponential light decay.

\subsection{Background}

\subsubsection{Theoretical Background}

The main research objective to tackle is experimentally demonstrating whether there is a non-trivial, and measurable change in light decay within a turbulent cloud, due to mixing and cloud in-homogeneities. Under turbulent conditions there could exist non-poissonian conditions for droplet spatial distribution, and if strong enough, it may be that the exponential decay of light described by the Beer-Lambert law does not apply. This is due to the lack of perfectly random distribution, due to the presence of turbulence. From the paper "On the extinction of radiation by homogeneous but 
spatially correlated random medium" Kostinski, 2001) the author establishes the connection between a stochastic distribution of cloud particles (Poisson distributed) and exponential light decay of light from the Beer-Lambert law using the following equations:

$$
\begin{gathered}
p_{n}(x)=\frac{\left.\left(\overline{\left(n(x)^{n}\right)} \exp [-\overline{(n(x)})\right]\right)}{n !} \\
p_{0}(x)=\frac{N_{t r}}{N_{\text {inc }}}=\exp [-\overline{(n(x))}]=\exp (-\beta x)
\end{gathered}
$$

Where $p_{n}(x)$ "is the probability of having $\mathrm{n}$ photons absorbed in a given volume of a layer of depth $\mathrm{x}$, and $\overline{n(x)}$ is the mean count over many realizations as a function of the depth $\mathrm{x}$ into the slab." Kostinski, 2001) and the " $N$ stands for the number of incident and transmitted photons... and $\beta x$ is the optical depth" Kostinski, 2001). The above equation operates under the assumption that, when particles are categorized into different layers, their spatial location are statistically independent from each other. The author then relaxes the assumption and reinterprets equation (1.2), and results in equation (1.3):

$$
p_{0}(x)=\frac{N_{t r}}{N_{\text {inc }}}=\frac{1}{1+\beta x}
$$

Which represents a probably of zero photons being absorbed by coming into contact 
with a particle. The key difference here is that for any value of $\beta x$, the overall probability of zero photons coming into contact and being absorbed by a particle is smaller when the assumption of spatial independence is taken out. Meaning that for a given DSD, with a calculated optical depth $\beta x$ the actual probability of a photon coming into contact with a droplet and being absorb changes depending on the correlation between droplets. And while the author primarily emphasized extinction via absorption throughout the paper, the logic also applies to extinction via scattering. This brings up questions as to how differently correlated media would act in real meteorological and turbulent environments. And if turbulence can serve as a correlation parameter for the spatial distribution of non-overlapping layers. The above equations serve as an inspiration for this research endeavor.

\subsubsection{Computational/Experimental Background}

This experiment is further inspired by the paper "Super-exponential extinction of radiation in a negatively correlated random medium" (Shaw et al., 2002$)$, where Monte Carlo simulation was used to represent uniform and non-uniformly correlated particle placement within a virtual atmosphere. Through this simulation they tested for both positively correlated and negatively correlated particle positions. As shown in figure (1.1), positive correlation within the medium meant that more radiative intensity goes through, instead of the exponential extinction predicted by the Beer-Lambert 
law, the extinction is sub-exponential. Also from figure (1.1), we can expect that if there exists a deviation for the OD due to in-homogeneous correlation is particles, it is much more likely to result in an increase of the mean free path of light.

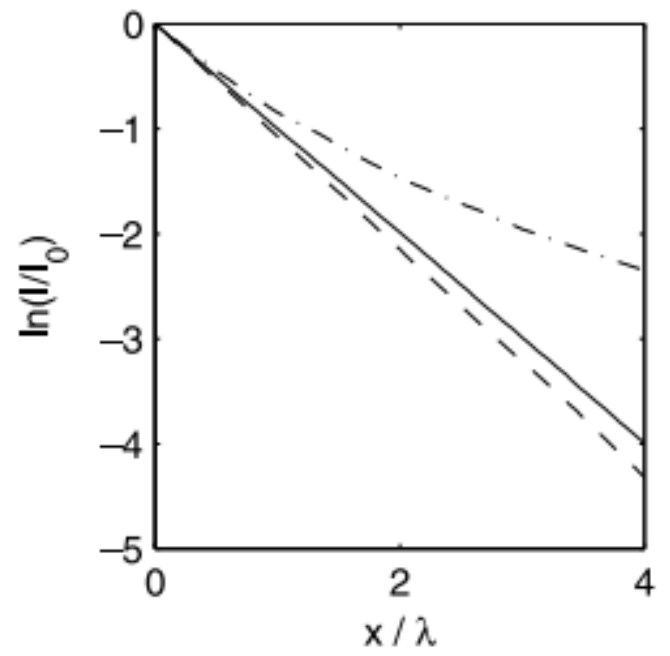

Figure 1.1: Relative intensity of light as a function of propagation distance. Where the solid line is from a uniform medium and in agreement with BeerLambert. Top line is for positively correlated non-uniform medium, and lower line is for negatively correlated non-uniform medium (Shaw et al. 2002). Permission was granted by JQSRT for use this figure.

In figure (1.2) we show an example of how scattering takes place in a Monte Carlo simulation, where photons are shot at a collection of particles and then scattered accordingly in different directions depending on the probabilities derived from the size and refractive index of the particle. The simulation shown in figure (1.2) is for the forward scattering geometry inside the $\pi$ - Chamber. The aim of our research is to experimentally explore what has been previously simulated, determining whether conditions exist that produce a nontrivial departure from exponential light decay. The 
goal is to connect that to the spatial distribution of non-uniform cloud environments created by turbulent conditions, which could then be extended to an atmospheric context. Previous works have already tackled means on how to computationally witness the presence of light intensity deviation from the expected results of a noncorrelated homogeneous scenario, (Davis and Mineev-Weinstein, 2011; Larsen and Clark, 2014; Frankel et al., 2016). In particular, Frankel et al. (2017) showed that if DSD and flow parameters are known, then an appropriate correction term can be applied to the of light. Other published literature has looked into experimental means of seeing the effects of turbulence on light propagation (Pawar and Arakeri 2016; Yuan et al., 2014, Consortini et al., 1980; Packard et al., 2018).

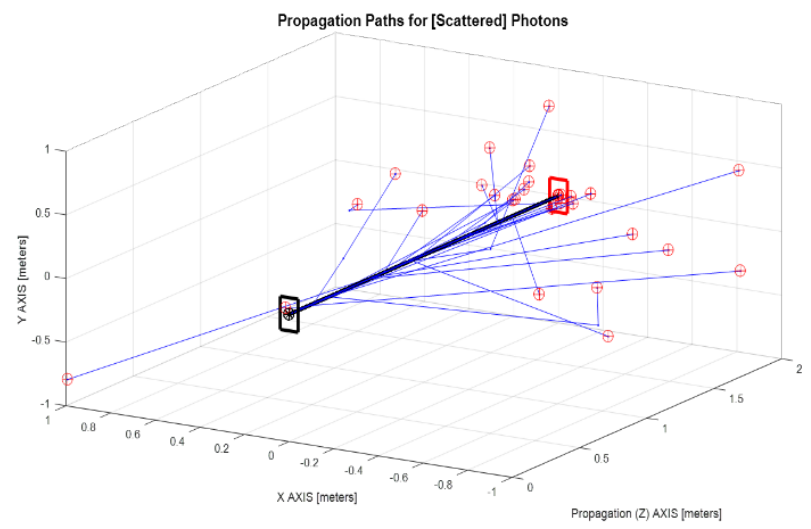

Figure 1.2: An example of light scattering in a Monte Carlo simulation. Image provided by Corey Packard. Note that most of the particles are scattered in the forward direction with a very small deviation from the center. 


\subsection{Experiment and Equipment}

\subsubsection{Experiment}

Our research is meant to build upon the previous computational and theoretical understanding of this Beer-Lambert deviation phenomena by creating an experimental representation of it. The experiment we perform is the comparison of a LaserHygrometer and a DSD analysis of the same turbulent cloud conditions created in side a cloud chamber. The Laser-Hygrometer will provide a physical transmission ratio of light intensity, while the DSD will be acquired at the same time. Through Beer-Lambert equation, an OD will be taken from the Laser-Hygrometer. While Mie scattering theory will be used for the DSD to obtain an overall extinction of light, in turn also obtaining the clouds OD. In an ideal scenario, where the spatial distribution of particles is non-correlated, both of this approaches to quantify OD inside a cloud chamber under turbulent conditions would yield similar results. But if previously stated research is to be correct (Kostinski, 2001), we will see a deviation of both results, and a presence of a positively, or negatively correlated medium for light propagation(Shaw et al., 2002). 


\subsubsection{Schematic}

The beam from the Laser-Hygrometer is collimated and split into two beams. One beam goes towards the the reference photo-diode(ai1), while the other will go towards the experimental photo-diode(ai0). The experimental photo-diode will be past the $\pi$-Chamber while the reference will be near the source of the Laser on the same side of the $\pi$-Chamber. A schematic of the above can be found in figure 1.3.

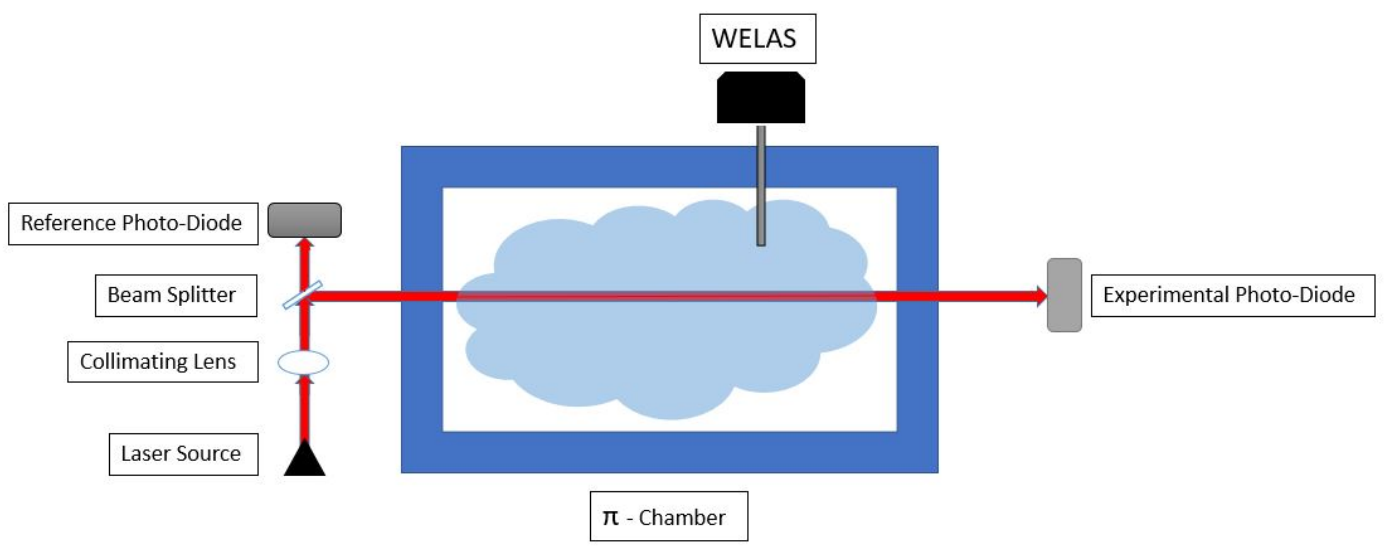

Figure 1.3: A schematic representation of the experimental setup for the Laser-Hygrometer. Photo-Diode ai1 is the reference, and Photo-Diode ai0 is the experimental

\subsection{3 $\pi$-Chamber}

The primary piece of equipment to be used is the $\pi$-Chamber at Michigan Technological University. The $\pi$-Chamber is a cloud chamber with an internal operating 
volume of 5 cubic meters, but after the inner cylinder has been inserted the volume reduces to of 3.14 cubic meters (Chang et al. 2016 ). The cylinder effectively creates two distinguished sections inside of the $\pi$-Chamber, which can hold different properties, such as RH\% and droplet concentrations. Some of the controlled properties include pressure, temperature difference between the floor and ceiling, and number of aerosol nucleation sites. Pressure inside can be set to lower than local surface pressure conditions at a rate of $-220 \mathrm{Pas}^{-1}$, down to $60 \mathrm{hPa}$ (Stephens et al., 2018). While the temperature of the floor and ceiling has a range from $-55^{\circ} \mathrm{C}$ to $55^{\circ} \mathrm{C}$. These parameters allow for the creation of two types of clouds, expansion and steady-state clouds (Stephens et al., 2018). Our research focuses on steady-state conditions inside the cloud chamber pertaining to particle gain and loss inside the $\pi$-Chamber. The aerosol input used is in the order of $\approx 10^{6} \# / \mathrm{cm}^{3}$ per 132 seconds. When particle equilibrium is reached the settling of particles and droplets inside the chamber equals that of the influx. This conditions are ideal for the experiment since it means that we can create a controlled environment, with steady turbulence and nucleation sites. This allows us to observe how turbulence affect droplet size, uniformity, and in turn light extinction. In figure (1.3) we show that droplets are not distributed uniformly due to the presence of small eddies. 


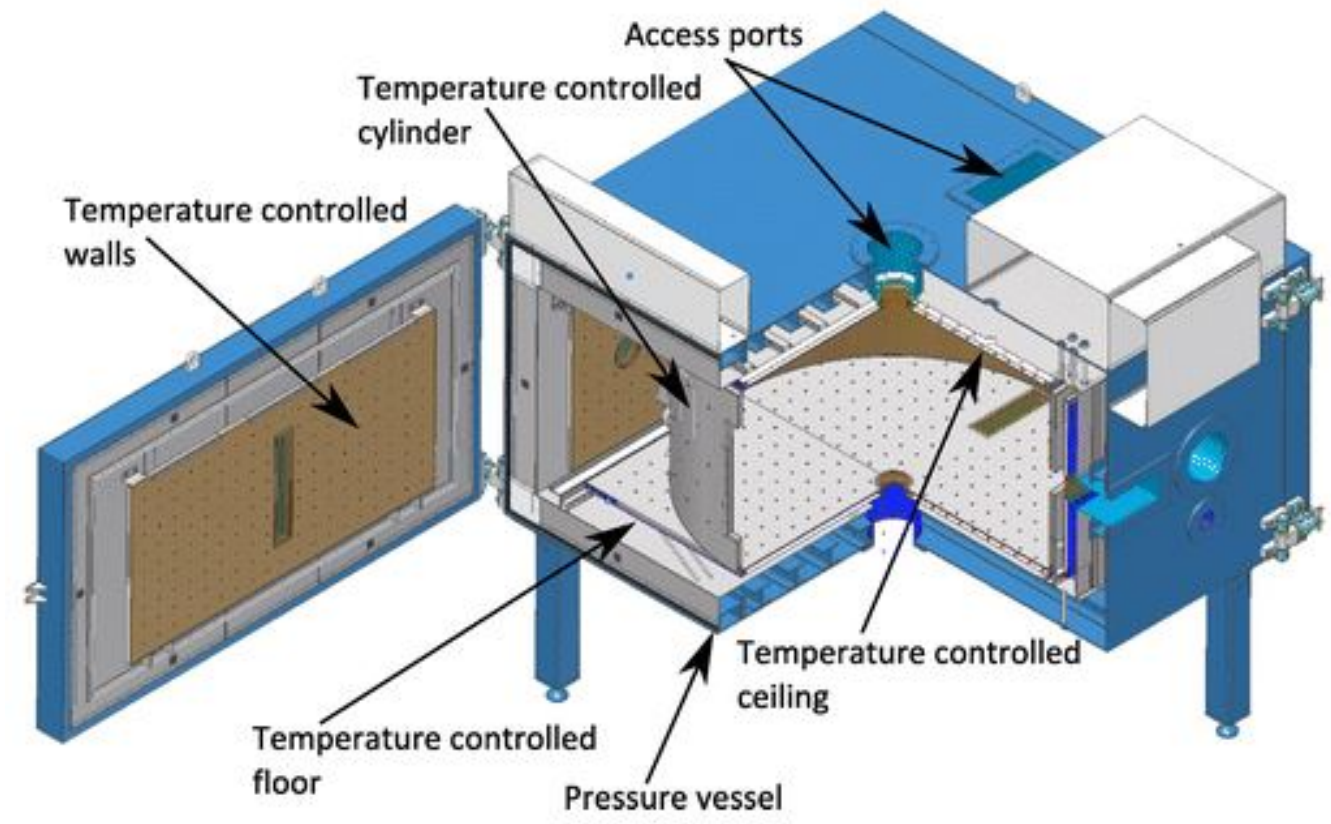

(a) Schematic view of the $\pi$-Chamber from (Chandrakar et al., 2016). Permission was granted by AMS to use this figure.

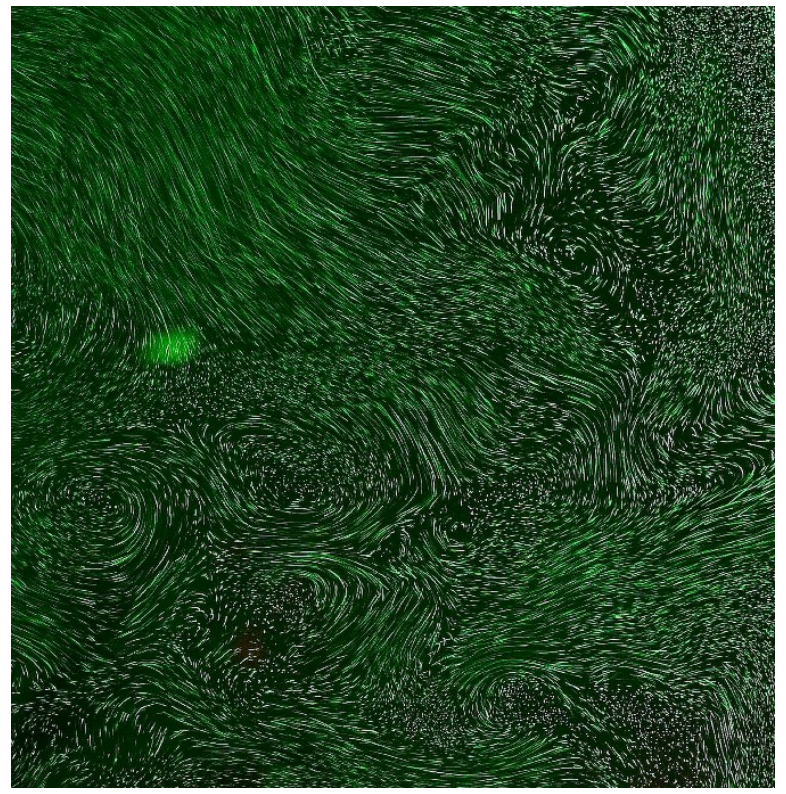

(b) Cross-Section of a turbulent Cloud Inside the Chamber, Image provided by Kamal Kant Chandrakar

Figure 1.4: $\pi$-Chamber 


\subsubsection{Laser and Photo-Diodes}

The laser utilized in the experiment is a DX1 laser diode from (eblanaphotonics), a distributed feedback tune-able laser. By controlling the $V_{\text {bias }}$ and $V_{\text {tec }}$ the laser is able to scan the wavelength range of $1378 \mathrm{~nm}$ to $1400 \mathrm{~nm}$. The laser operates with a near monochromatic line-width frequency of $2 \mathrm{MHz}$. In our Area of Interest(AoI), which corresponds to a water vapor absorption bands, of $1392 \mathrm{~nm}$ there exists a wavelength line-width of the laser is $1.29 \times 10^{-5} \mathrm{~nm}$. Such low deviation allows us to classify the laser as near monochromatic for the purpose of the experiment, in which we only consider wavelength resolution down to the scale of $1 \times 10^{-2} \mathrm{~nm}$. The laser is designed to operate in a wavelength range particularly sensitive to $\mathrm{H}_{2} \mathrm{O}$ vapor absorption bands. The photo-diodes in use are a SM05PD6A from (Thorlabs $)$ which are sensitive to the NIR range of 800 - $1800 \mathrm{~nm}$ wavelength, they are also equipped with an NENIR10B-C and NENIR20B-C neutral density filters from Thorlabs. Lastly an aluminum block of comparable size to the laser block is attached to the laser to serve as a heat-sink and stabilize the monochromatic wavelength out put as it scans the wavelength range. An example of the arrangement of the laser and diode can be found in figure (1.5) 


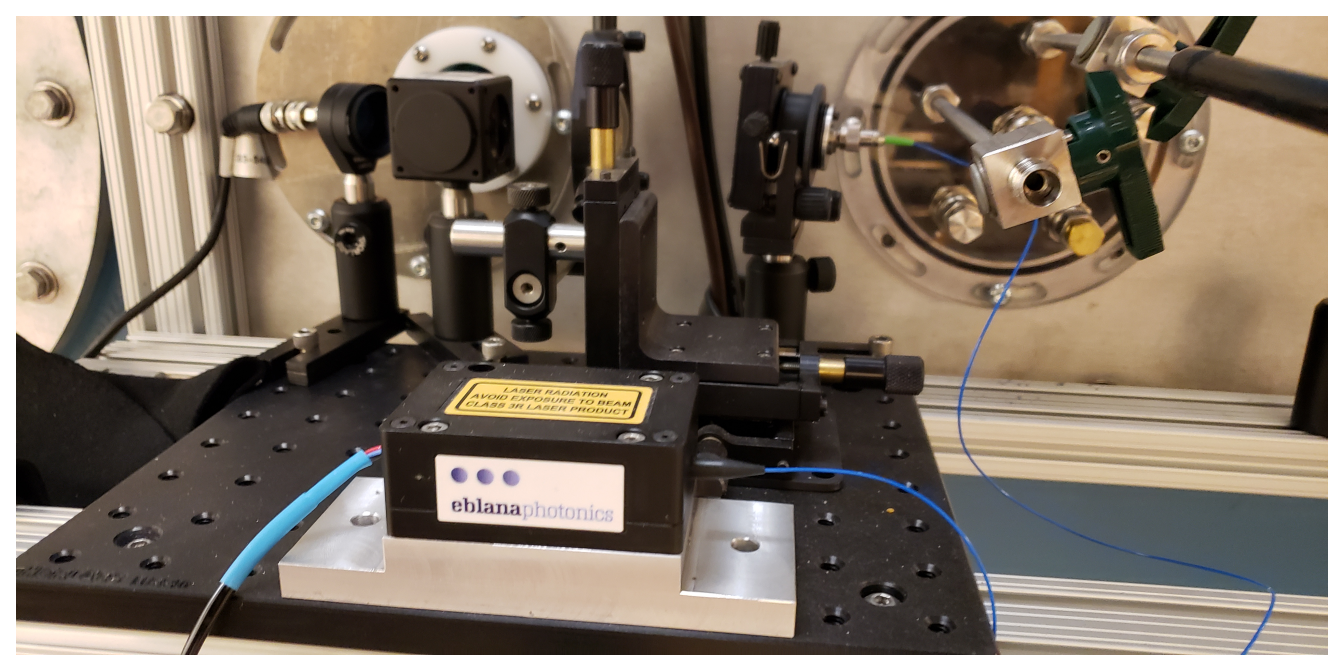

(a) Laser and Reference Photo-diode

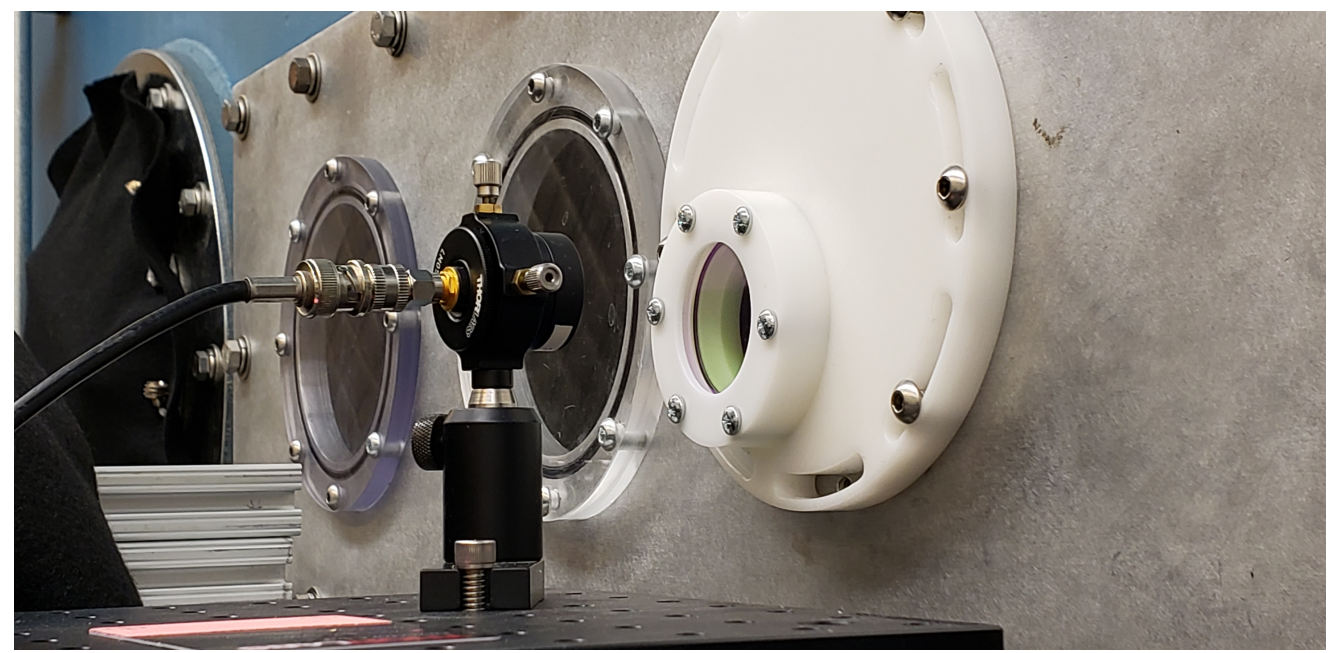

(b) Experimental Photo-diode

Figure 1.5: Pictures of the optical set-up including the NIR Laser and Photo-diodes. The laser beam passes through to outside windows, NBK-7 2 "diameter and $12 \mathrm{~mm}$ thickness windows with the $\mathrm{C}$ coating. And two inner windows, made up of float glass of 3/16 thickness. All windows were chosen because of their low absorption and high transmission rates around $1392 \mathrm{~nm}$ wavelength range. 


\subsubsection{Data Acquisition}

The data acquisition card in use was the NI PCI-6110 card. Its main attributes that contributed towards the research was its capabilities for simultaneous analog input and output capabilities. Analog output range is \pm 10 with an absolute accuracy of $0.0018 \%$ and maximum offset of $5.933 \mathrm{mV}$. Analog input range is $\pm 42 \mathrm{~V}$, within our desired area of operation $\pm 0.5 \mathrm{~V}$ the absolute accuracy ready is $0.057 \%$ and a maximum off set of $0.4 \mathrm{mV}$. Finally, the operating frequency range is $10 \mathrm{MHz}$ with a base clock accuracy of $0.01 \%$. $(N I, 2004)$. The PCI-6110 is used to send the operating voltage signal to the Laser-Hygrometer and to receive the input voltage from the two photo-diodes.

\subsubsection{WELAS}

The WELAS digital 200 series is capable of measure and cataloguing particle size distributions in the size ranges of $0.2-10 \mu m$ and $0.6-40 \mu m$. With this instrument we are able to acquire concentrations of specific droplets size with a given volume, ranging from $1 \# / \mathrm{cm}^{3}$ to $10^{6} \# / \mathrm{cm}^{3}$. The WELAS takes in air through a pipe inside the chamber. Particles, such as aerosols and droplets, are analyzed by having white light shot at them. The scattered light at $90^{\circ}$ is used to determine the characteristic 
particle size. Factors such as the velocity, refractive index, and amplitude factor are taken into account when determining the size of the transient particle. Processing rate of the WELAS is $20 \mathrm{MHz}$ which allows it to analyze the progression of each particle signal. $(P A L A S)$ 



\section{Chapter 2}

\section{Procedures \& Experiment}

\subsection{Procedures for Relative Humidity Readings}

The first step for the experiment is more of a sanity check. We wish to use the tuning laser in order to get an appropriate $\mathrm{RH} \%$ reading that matches our expected values inside the $\pi$-Chamber. This is a valuable check because the $\mathrm{RH} \%$ readings is going to be based of the voltage differences at specific $\mathrm{H}_{2} \mathrm{O}$ vapor absorption lines.

Beginning with the tuning laser, we shift the voltage bias through a range of $0.2 \mathrm{~V}$ to $1.2 \mathrm{~V}$ and scan through a wavelength range of $1392.2 \mathrm{~nm}$ to $1392.8 \mathrm{~nm}$. Within this range we look at specific water vapor absorption lines to determine the overall OD caused by absorption within the chamber in moist conditions without cloud droplets. 
This is done in order to derive the molecular concentration of water vapor and its overall impact in light absorption at a given wavelength. We obtain the water vapor concentration and OD from measuring the light intensity difference before, using the reference photo-diode, and after, using the experimental photo-diode, the laser beam travels through the chamber. In figure (2.1) we show an example of how the light intensity difference, in units of voltage is measure between both photo-diodes at the same wave-number. Subsequent calculation relies on the use of known cross-sectional absorption of water vapor obtained from the HITRAN database (Gordon et al., 2017), and the following equations

$$
\begin{gathered}
N=\frac{-\ln \left(\frac{\phi_{F}}{\phi_{I}}\right)}{\left(X S_{a b s} \times L\right)} \\
P_{p}=\frac{N}{(N a)} \times(R \times T)
\end{gathered}
$$

Where $N$ is the molecular concentration $\left(\right.$ molecule $\left./ \mathrm{cm}^{3}\right), \phi_{I}$ and $\phi_{F}$ are the initial(ai1) and final(ai0) radiant power $(W$, but readings are in $V$ ) readings after transmitting through the $\pi$-Chamber, $X S_{a b s}$ is the cross-section efficiency of water vapor $\left(\mathrm{cm}^{2} /\right.$ molecule) obtained from HITRAN Gordon et al., 2017) plotted in figure (2.2), $L$ is the length of the chamber $(\mathrm{cm})$. Equation $(2.2)$ is the ideal gas law and $P_{p}$ is the partial pressure by water vapor, $N a$ is Avogadros number (molecules/mol), $R$ is the gas Constant $\left(\mathrm{cm}^{3} \mathrm{kPa} /(\mathrm{Kmol})\right)$, and $T$ is the temperature $(K)$. With this equations we know the partial pressure of water vapor inside the chamber. We then found the 
equilibrium pressure of water above a liquid layer using the following equations.

$$
\begin{gathered}
P_{w}=0.61121 \times \exp \left(\left(18.678-\frac{T_{c}}{234.5}\right) \times\left(\frac{T_{c}}{257.14+T_{c}}\right)\right) \\
R H \%=\frac{P_{p}}{P_{w}} \times 100
\end{gathered}
$$

Equation (2.3) is the Arden Buck equation derived in $(B u c k, 1981) . P_{w}$ is the equilibrium pressure of water vapor for a known temperature $(k P a), T_{c}$ is chamber temperature in celsius which is taken from the $\pi$-Chamber computer. $R H \%$ is the relative humidity.
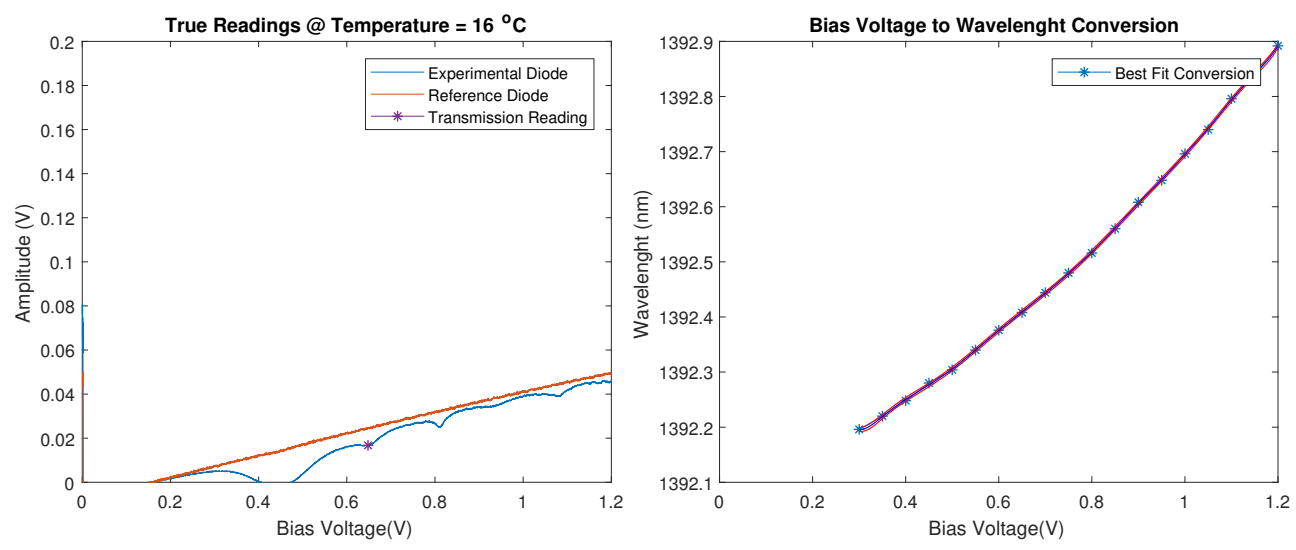

Figure 2.1: An example of how data is acquired using the LaserHygrometer. The first plot shows the true readings from both photo-diodes as it scans the AoI by changin the $V_{\text {bias }}$. Second plot is the wavelength output of the laser at a given $V_{\text {bias }}$ for a $V_{\text {tec }}$ of $0.4 \mathrm{~V}$. The last plot represents the converted values of voltage at specific wave-numbers, centered about the point which we focus on the transmission rate

The initial step of this research is to make sure that the equipment is measuring what we expect it to measure. As a form of evaluating the validity of the procedure, we 


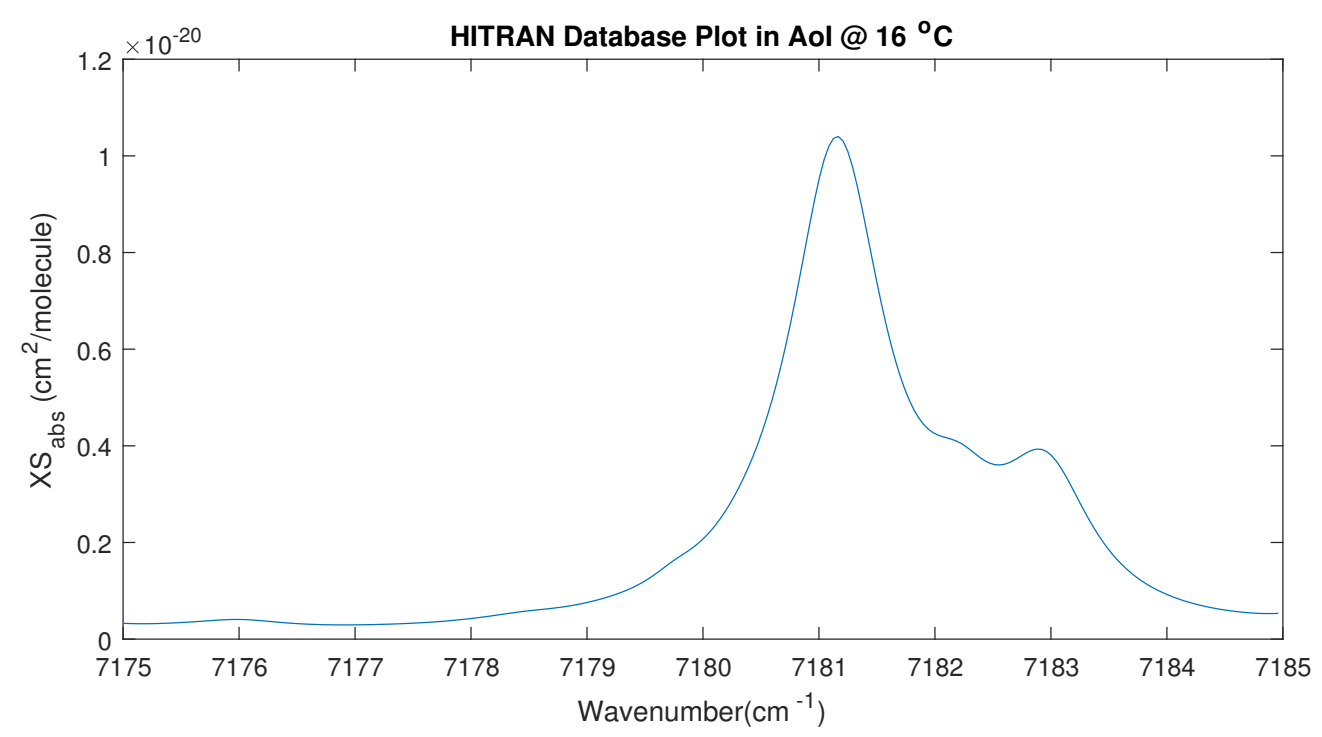

Figure 2.2: The above plot shows the water vapor absorption lines in the AoI. The $X S_{a b s}$ values of water at a given wave-number are extracted for further analysis into the $\mathrm{RH}$ inside the $\pi$-Chamber. The data that the plot consist of was retrieved from the HITRAN database.

first use the previous equations to analyze data taken in an environment inside the cloud chamber where there are no water droplets, at a relative humidity of $<100 \%$. If the transmission ratio is correct, then the $P_{P}$ can be used to then find the $\mathrm{RH}$ inside the chamber. When the $\mathrm{RH} \%$ from the Laser-Hygrometer matches the $\mathrm{RH} \%$ readings of the $\pi$-Chamber computer in the range near $100 \%$ but below super saturation, we will know that the transmission readings are accurate.

The main thing that have to be considered here is the path length that the laser beam must travel. Due to the presence of the cylinder and window indentations inside the chamber, the humidity is not uniform through the $\approx 243 \mathrm{~cm}$ path and is likely to be far higher within the cylinder then outside of it. Because of of this we expect calculations based on $200 \mathrm{~cm}$, the path length of the cylinder inside the $\pi$-Chamber, 
to over estimate. While calculations based on $243 \mathrm{~cm}$, the full path length of the $\pi$-Chamber, to under estimate the $\mathrm{RH} \%$ readings.

\subsubsection{Optical Depth Readings, Laser-Hygrometer}

The OD readings from the Laser-Hygrometer come from the the voltage ratio detected by the reference, and experimental photo-diode. The Laser is set to tune across a wavelength range about $1392 \mathrm{~nm}$ as shown in figure (2.1). In order to account for the transmittal differences due to travel distance, slight extinction due to windows, and unequal intensity distribution at the beam splitter, an adjusting scale factor is applied to the reference voltage to match the experimental where there is no extinction due to humidity or cloud conditions. The scaling factor is usually withing the range of 0.9 1.1. Within the tuning range the MATLAB code for data acquisition is set up so that it finds a specific maxima that corresponds to a water absorption band. This is done so that we know for certain that we are still operating on the same wavelength and that external factors such as heat build up in the laser has not shifted the relationship between $V_{b}$ and its corresponding wavelength. The voltage difference is taken between the photo-diodes and used to calculate a direct OD using the Beer-Lambert Law using formula found in (Petty, 1998)

$$
\frac{\phi_{F}}{\phi_{I}}=e^{-\tau}
$$




$$
\tau=-\ln \left(\frac{\phi_{F}}{\phi_{I}}\right)
$$

Where $\tau$ is the optical depth (OD). A timed average value is taken at different intervals to be used for cross comparing with other data sets. The light extinction caused by the presence of water vapor is subtracted from the overall extinction, leaving behind only the extinction caused by the scattering from the water droplets.

\subsubsection{Optical Depth Readings, WELAS}

Using the WELAS we can obtain a DSD near the the middle of the $\pi$-Chamber, where the Laser-Hygrometer beam passes. This DSD is used in conjunction with Mie Scattering code. The Mie Scattering MATLAB code is an altered version of the code published by Christian Matzler (Matzler, 2002). The computed values for extinction, scattering, and absorption are checked and validated by comparing to multiple published works (Shah, 1977), Wiscombe, 1979), (Du, 2004), Wang and van de Hulst, 1991) for different size parameters and refractive index's. The output from the WELAS provides droplet diameters in concentration bins. The MATLAB code computes the extinction coefficient via Mie Theory. The extinction coefficient is obtained by adding together the Scattering efficiency, which is dominant, and the absorption coefficient, which is orders of magnitude smaller and insignificant, of the droplet at the given size parameter $X$ (defined in equation (2.7)) and refractive index. The 
refractive index we used is $1.3211+i 1.3 \times 10^{-4}$ (Hale and Querry, 1973). Note that this is the refractive index for water for an incident wavelength of $1392 \mu m$ and a temperature of $25^{\circ} \mathrm{C}$, which is not the same temperature that we are working in. The size parameter $X$ is obtained using the following definition retrieved from Bohren and Huffman, 1998),

$$
X=\frac{2 \pi R}{\lambda}
$$

Where the $\lambda$ is the wavelength $(\mu m)$ used by the Laser-Hygrometer, and $R(\mu m)$ is the radius of the specific bin from the DSD. When the extinction coefficient of the specific droplet size is found, we then multiply it by it's respective cross sectional area and concentration, given by the WELAS. We also take into consideration the forward scattered percentage of photons that after being scattered by a particle, are still in the field of view and might be detected by the experimental photo-diode(ai0), and thus would be erroneously considered as not contributing to the overall extinction in our experiment. Using the following summation found in Petty, 1998 for all the bins provides us with the OD inside the $\pi$-Chamber via computational means

$$
\tau=\sum \sigma R^{2} \pi l \times \rho \alpha
$$


Where the $\sigma$ is the extinction coefficient, $l$ is the path length inside the cylinder inside the $\pi$-Chamber of $\approx 2 m, \rho$ is the concentration of the particle of a specific size in $\# / m^{3}$, and $\alpha$ is the forward-scattering angle efficiency parameter. The forward-scattering efficiency parameter is determined by finding the angular fraction, which changes depending on location we interpret the cloud droplet to be. The forward scattering efficiency is found by using (Bohren and Huffman, 1998)

$$
\alpha=1-\frac{2 \pi \int_{\theta_{p}} Y(\theta) \sin (\theta) d \theta}{2 \pi \int_{\theta_{f}} Y(\theta) \sin (\theta) d \theta},
$$

where $\theta_{p}=\arctan (a / d), \theta_{f}$ represents the full $180^{\circ}(\pi)$ range of half the polar plot, $Y(\theta)$ is the amplitude of the scattering angle dependence, $a$ is the radius (3mm) of the photo-diode sensor, and $d$ is the distance from the droplet to the experimental photo-diode, which varies from $0.2 \mathrm{~m}$ to $2.2 \mathrm{~m}$. The effects of the forward scattering are visualized on polar plots in figure (2.3).

When taking into account the efficiency for forward scattering for each of the size parameters, 116 bins in total, we get the plot shown in figure (2.4) where the efficiency of water droplets at different distances inside the $\pi$-Chamber are shown.

The DSD from the WELAS is collected during steady-state conditions when temperature differences between the ceiling and floor are applied. The aerosol input is 2.6 million per cubic centimeter. This large influx was chosen due to the need to create 


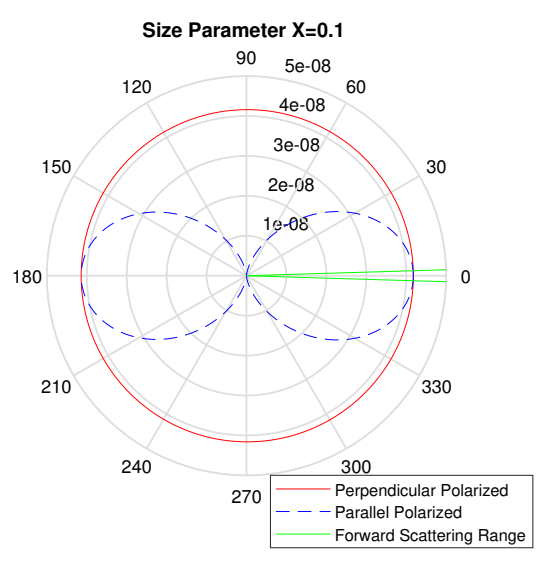

(a) $\mathrm{x}=0.1$

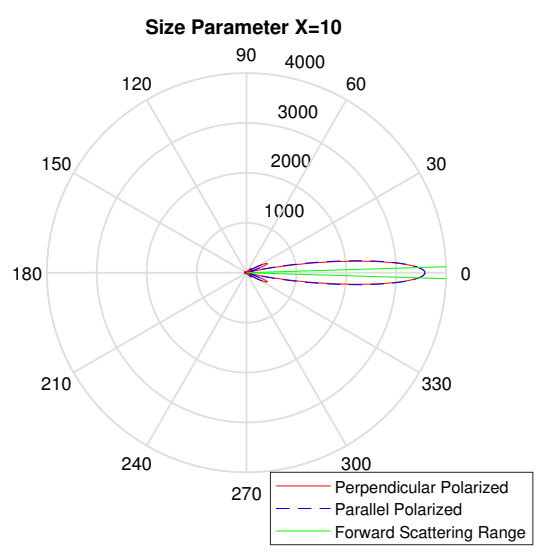

(c) $\mathrm{x}=10$

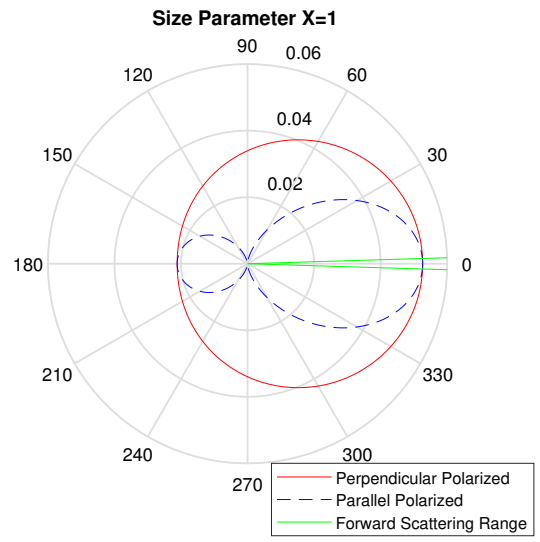

(b) $\mathrm{x}=1$

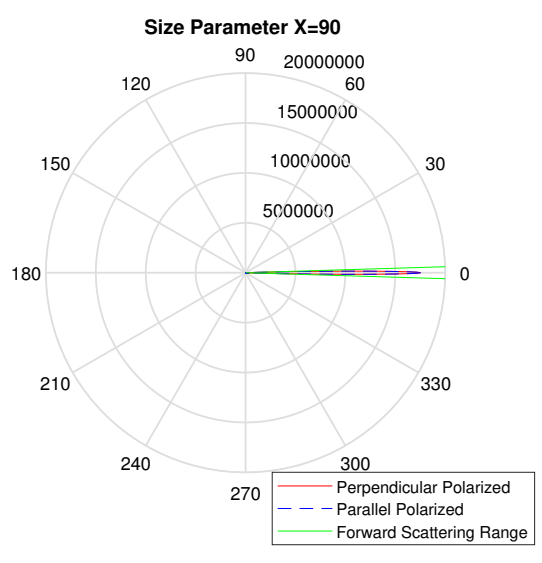

(d) $\mathrm{x}=90$

Figure 2.3: The scattering angle dependence of light as it comes into contact with different sized particles, at a refractive index of 1.33. Additionally, the forward scattering angle deficiency is shown for the case where the particle is located $\approx 2.2 \mathrm{~m}$ away from the experimental photo-diode. The plots were created using the code from Christian Matzler (Matzler, 2002). The size parameters chosen nearly correspond to actual size parameters calculated from the DSD. In terms of droplet radius the polar plots correspond to (a) $\mathrm{R}=0.022 \mu m$,(b) $\mathrm{R}=0.22 \mu m$, (c) $\mathrm{R}=2.2 \mu m$, (d) $\mathrm{R}=19.9 \mu \mathrm{m}$. Note that as the size parameter increases, the ratio of area within the forward scatting deficiency, relative to the area outside of it, increases.

significantly large OD through the Twomey effect (Twomey, 1974). It was also necessary to keep droplet sizes within the smaller detectable range of the WELAS, and 


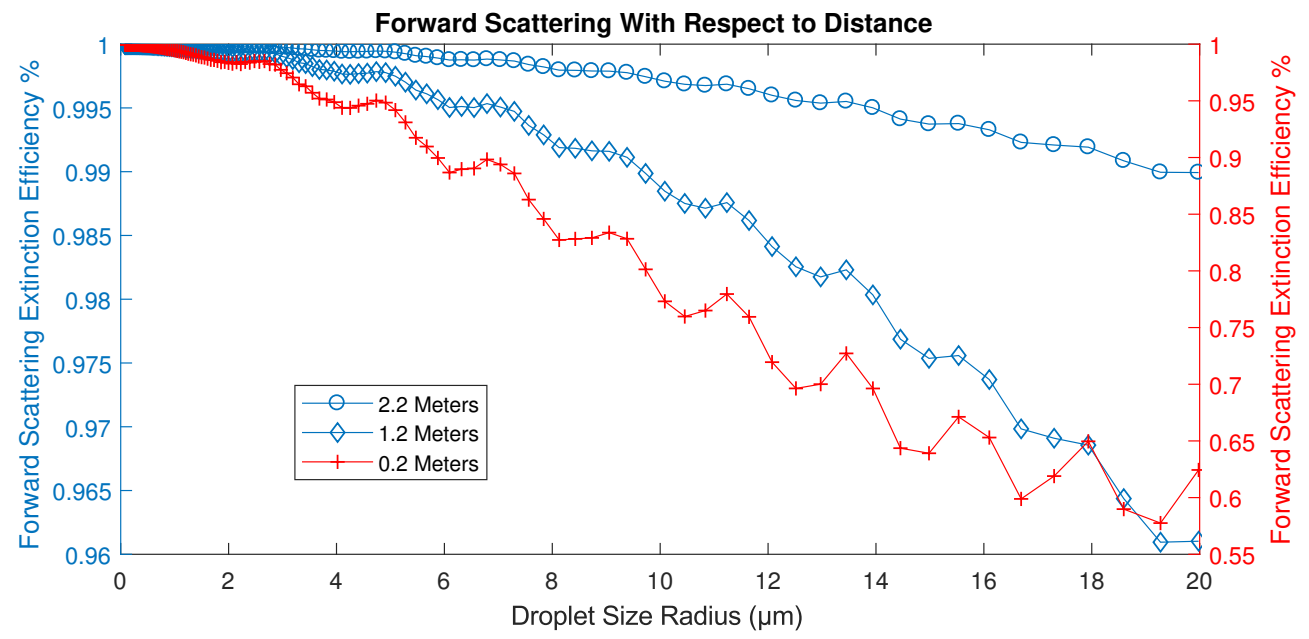

Figure 2.4: The forward scattering efficiency for three different distances with respect to the experimental photo-diode inside the $\pi$-Chamber, $2.2 \mathrm{~m}$, $1.2 \mathrm{~m}, 0.2 \mathrm{~m}$.

in turn not having to rely on other instruments that are more capable at cataloguing larger droplet sizes. One example of the of such instrument is the Phase-Doppler Interferometer (PDI), which is ineffective in detecting droplet sizes smaller than $10 \mu m$. In such a scenario where both the WALES and the PDI were to be used, it would require further effort and complications when combining the two DSD data sets into one.

\subsubsection{Optical Depth Comparison}

In order to create a turbulent conditions for both the WELAS and the LaserHygrometer to operate in, a temperature difference between the floor and ceiling is applied. The floor being set to a higher temperature, while the room is set to a lower 
temperature. This is done for the temperature differences of $5 \mathrm{~K}, 10 \mathrm{~K}, 15 \mathrm{~K}$, and $20 \mathrm{~K}$. When a new temperature difference is set on the $\pi$-Chamber there is a 45 -minutes to an hour wait time for the chamber to reach steady state.The Laser-Hygrometer runs continuously through tout the whole experiment. While the DSD is collected at three different points in time. Following the completion of one experiment, a new gradi$\operatorname{ent}(\mathrm{K} / \mathrm{m})$ is applied shortly after. This systematic approach allowed us to acquire the DSD at different gradients $(\mathrm{K} / \mathrm{m})$ about the same mean temperature $\left(10^{\circ}\right)$. When the OD for both the WELAS and Laser-Hygrometer are computed and measured for each of the four gradients $(\mathrm{K} / \mathrm{m})$, we plot them together and analyze the content. The uncertainty of the WELAS comes from our lack of knowledge as to the position of the droplet withing the cylinder, which greatly determines the forward scattering efficiency. The center distance inside cylinder will is interpreted as the mean location of the droplet, which corresponds to $1.2 \mathrm{~m}$. While the lower uncertainty is for $2.2 \mathrm{~m}$ and the upper uncertainty is for $0.2 \mathrm{~m}$. 



\section{Chapter 3}

\section{Results and Discussion}

\section{$3.1 \quad$ Overview}

In this section we will go over the main parts of the research and their results. We will start with relative humidity readings inside the $\pi$-Chamber which told us whether the method used for interpreting transmission through intensity ratio between the photodiodes is correct and sufficiently accurate to proceed further. Afterwards we look at the results for the computational OD, from the WELAS DSD and Mie theory, and the experimental OD, from the Laser-Hygrometer. Lastly we compare the two OD values to record if and how they differ. 


\subsection{Relative Humidity Results}

The $R H \%$ was measured using the Laser-Hygrometer alongside the HITRAN data (Gordon et al., 2017), from which we got the results shown in figure (3.1).The condition inside the $\pi$-Chamber were that of a mean temperature of $11^{\circ} \mathrm{C}$ with no temperature difference between the floor and ceiling.

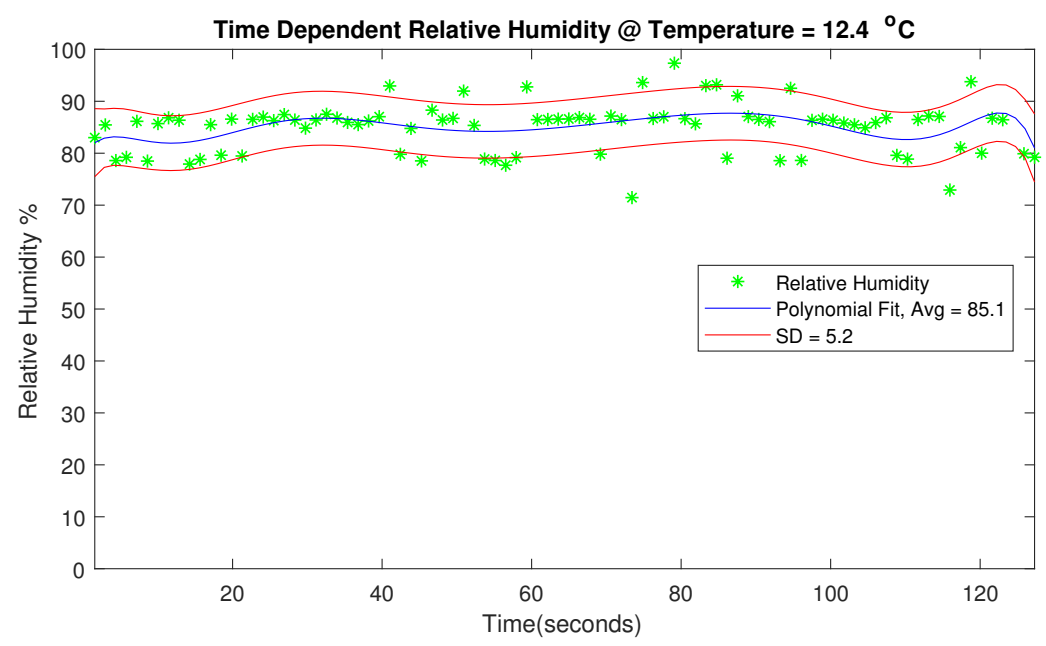

Figure 3.1: Relative humidity readings inside the $\pi$-Chamber, The chamber computer was reading an $R H \%$ of $88 \%$. The $3 \%$ discrepancy corresponds to an OD difference of 0.01 . This number was acquired by working backwards on equations (2.1)-(2.4). Blue line represent a poly-fitted line of the $10^{\text {th }}$ order degree. While the red-line is one standard deviation from it, both above and below.

From figure (3.1) we can be confident that the transmission readings of the LaserHygrometer are accurate, and in turn the OD readings will be a as well. Conditions inside the chamber would have suggested that without the presence of a temperature difference the $R H \%$ inside would be more steady. This may imply that the precision 
of the algorithm used to measure the light difference could still be improved. Additionally it could also mean that conditions inside the Chambers are rarely steady even under ideal circumstances as seen by the existence of some variations on the $R H \%$ levels. With it we accomplished the first step of this experiment where we determine if the transmissions rates derived from the difference between the diodes is accurate enough to give us a $R H \%$ reading.

\subsection{WELAS and Mie Scattering Optical Depth}

\subsubsection{Results}

Following the procedure previously stated in Chapter 2 for the OD of DSD we acquired the following data shown in figure (3.1). WELAS data was recording alongside the Laser-Hygrometer. The WELAS was ran under two settings. First setting collected DSD in the $0.2-10 \mu m$ diameter range, while the second setting collected a DSD in the 0.6-40 $\mu \mathrm{m}$ diameter range. The data below is acquired by splicing together the WELAS data from the two different DSD readings. It was spliced together at the point where the size bin was $0.898 \mu \mathrm{m}$ to get the a high resolution for both particles smaller and larger than $0.9 \mu \mathrm{m}$, which represent the hydrated aerosols and the activated droplets respectively. 


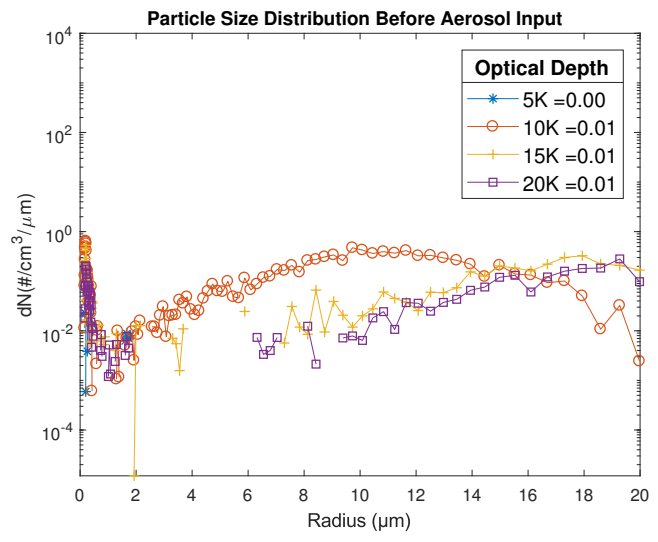

(a) Reference background particle size distribution

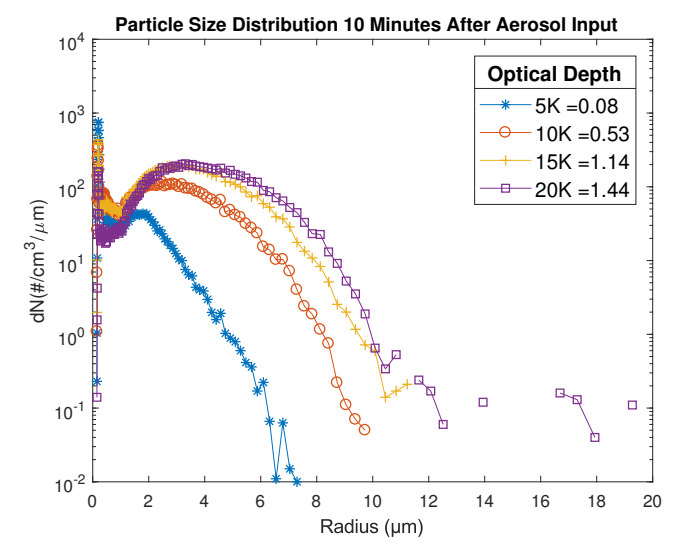

(b) Particle size distribution at $\approx 10$ minutes

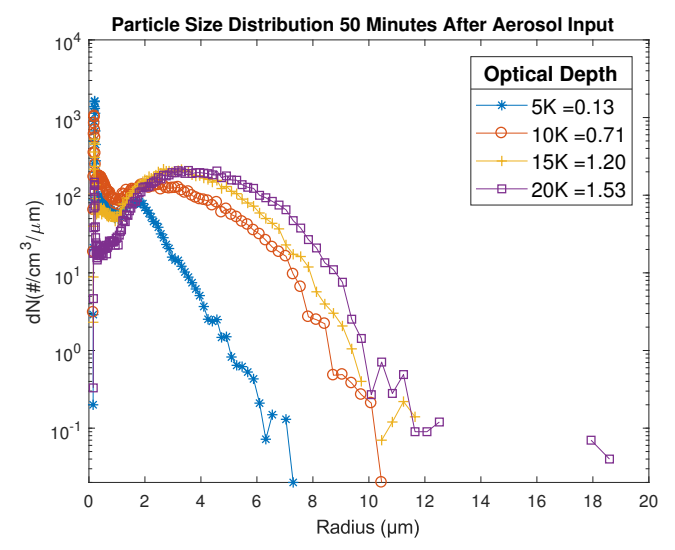

(c) Particle size distribution at $\approx 50$ minutes.

Figure 3.2: The particle size distribution inside the $\pi$ - Chamber at three different time periods. The bi-modal nature of the distribution is due to the presence of activated droplets and non-activated hydrated particles. The charts include the calculated OD of the size distribution. The Mie scattering calculations above were done under the scenario where the mean particle location was in the middle of the $\pi$ - Chamber, $1.2 \mathrm{~m}$ away from the experimental photo-diode.

The overall computed OD under the three different hypothetical scenarios for each distances from the experimental photo-diode $(2.2 m, 1.2 m$, and $0.2 m)$ are shown in table (3.1). The data below allow us to consider a uncertainty for the computed OD, where lower extinction is due to a close proximity to the experimental photo-diode 
and the higher extinction is due to a larger distance away from it.

\section{Table 3.1}

Overall Optical Depth at Different Distances, and Asymmetric Parameter

\begin{tabular}{r|rrrr}
\hline$\Delta K$ & $0.2 \mathrm{~m}$ & $1.2 \mathrm{~m}$ & $2.2 \mathrm{~m}$ & $\mathrm{G}$ \\
\hline $5 K$ & 0.13 & 0.13 & 0.13 & 0.33 \\
\hline $10 K$ & 0.68 & 0.72 & 0.72 & 0.50 \\
\hline $15 K$ & 1.13 & 1.20 & 1.20 & 0.60 \\
\hline $20 K$ & 1.43 & 1.53 & 1.53 & 0.71
\end{tabular}

\subsubsection{Analysis}

The background particle distribution for all four experiments show a very small concentration of particles and in-turn a very small contribution to the OD due to scattering. During the background example, the $\pi$ - Chamber is under supersaturated conditions, but is incapable of creating droplets due to the lack of nucleation sites, later provided by the $\mathrm{NaCl}$ aerosols. When the aerosols are included we can see that the higher gradients $(\mathrm{K} / \mathrm{m})$ are able to mix the cloud faster resulting in rapid creation of droplets. From figure (3.2) we see that at larger temperature differences, the OD rises faster, approaching its maximum steady-state OD sooner. This is shown by the difference of OD between the 10 minute and 50 minute marks for $20 \mathrm{~K} / \mathrm{m}$ gradient is only $\approx 0.8$ while the $5 \mathrm{~K} / \mathrm{m}$ gradient nearly doubles in the same time span.

Additionally from figure(3.2), the shape of the DSD shifts further to the right as the 
$\Delta T$ increases. This implies that mean droplet size is also increasing. The overall droplet concentration, $n$, increased in the $5 K$ case. Also, concentration stayed relatively stable between the $10 K, 15 K, 20 K$ cases. Overall, this is indicating an increase in liquid water content (LWC), making the Twomey effect is not as relevant since LWC is not constant in this scenario. With larger droplets present, the effective cross-section extinction rates increased, leading to the increased OD as temperature differences increased.

From table (3.1) we also see that distance from the experimental photo-diode plays a larger role in larger temperature differences due to the presence of larger droplets having greater forward scattering amplitude. This results in the difference between $0.2 \mathrm{~m}$ and $2.2 \mathrm{~m}$ being $\tau \approx 0.1$ for $\mathrm{OD}$, while the difference for $5 \mathrm{~K} / \mathrm{m}$ gradient is only $\tau \approx 0.02$.

Lastly, the possible effect of multiple scattering on the results is considered. This is of potential importance because multiple scattering can result in diffuse light being transmitted to the detector, in addition to the directly-transmitted beam. A full treatment of this effect depends on the angular scattering pattern of the cloud droplets, as well as the angle subtended by the detector itself. This is the topic of ongoing work and only a rough estimate of the effect will be considered here. Using 
two-stream theory Petty, 1998) the diffuse transmitted light can be calculated as

$$
T_{\text {diff }}=\frac{1}{1+(1-G) \tau}-\exp (-\tau)
$$

where $G$ is the asymmetry parameter. A $G$ value that approaches 1 could lead to occurrence of multiple scattering and therefore to further disagreement between the OD values of the Laser-Hygrometer and WELAS. The values of $G$ that we found are shown in Table (3.1). In this context, the equation predicts how much scattered light reaches the other side of the $\pi$-Chamber after multiple scattering. For our largest value of $\tau$ and $G$ in the 20 -K difference case we find that $T_{\text {diff }} \approx 0.47$, which equates to a reduction in $\mathrm{OD}$ of $\approx 0.75$. While it appears to be very significant, this estimate of the loss of OD due to multiple scattering does not take into account the small angle $\left(\theta_{p}\right)$ it must scatter to in order to be detected by the photo-diode. The area ratio of the photo-diode in the forward direction is of order $1-\cos \theta_{p} \approx \theta_{p}^{2} / 2$, so for $\theta_{p} \approx 3 \times 10^{-3}$ radians corresponding to the 3 -mm-radius photo-diode, and a working distance of 1 meter, we obtain an area ratio of approximately $4.5 \times 10^{-6}$. Thus the increase in overall light transmission seen by the experimental photo-diode should be quite small and the interpretation of the results is not gravely affected. 


\subsection{Laser - Hygrometer Optical Depth}

\subsubsection{Results}

Following the procedures stated in Chapter 2 for the Laser-Hygrometer, the results presented in figure (3.3) were acquired. Data was recorded alongside the WELAS to ensure that both approaches are looking at the same type of cloud. In table (3.2), we show the quantified relevant values of the Laser-Hygrometer results.

Table 3.2

OD before, and after aerosol influx. Also included is their combine standard deviation to serve as an uncertainty factor.

\begin{tabular}{r|rrr}
\hline$\Delta K$ & Before & After & SD \\
\hline $5 K$ & 0.15 & 0.38 & 0.03 \\
\hline $10 K$ & 0.13 & 0.67 & 0.04 \\
\hline $15 K$ & 0.12 & 1.09 & 0.05 \\
\hline $20 K$ & 0.12 & 1.17 & 0.07
\end{tabular}

\subsubsection{Analysis}

The values for the OD throughout the four temperature differences stayed relatively similar before the injection of aerosols. This is to be expected since the beginning phase should only contain gases, such as water vapor at near $100 \% R H$. With a 


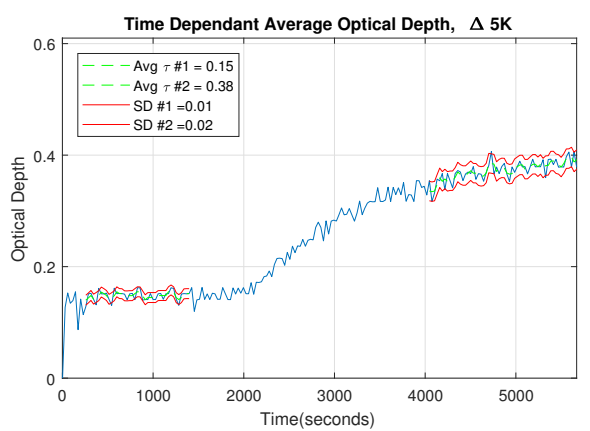

(a) Hygrometer Results at 5K/m Gradient

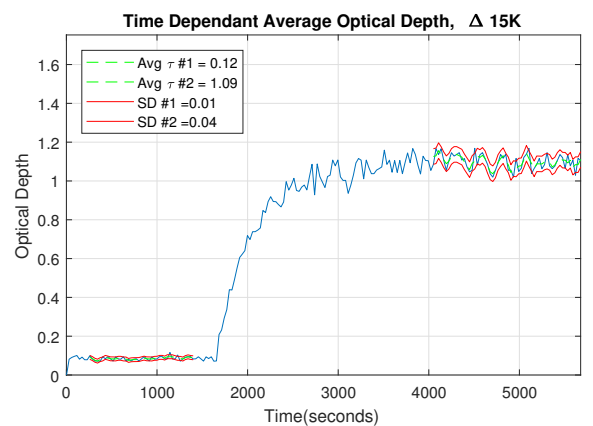

(c) Hygrometer Results at $15 \mathrm{~K} / \mathrm{m}$ Gradient

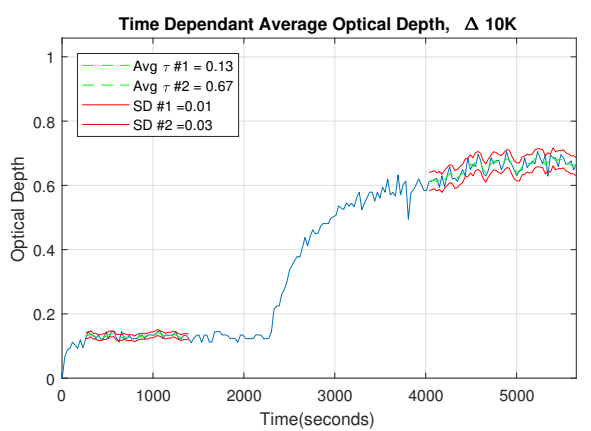

(b) Hygrometer Results at 10K/m Gradient

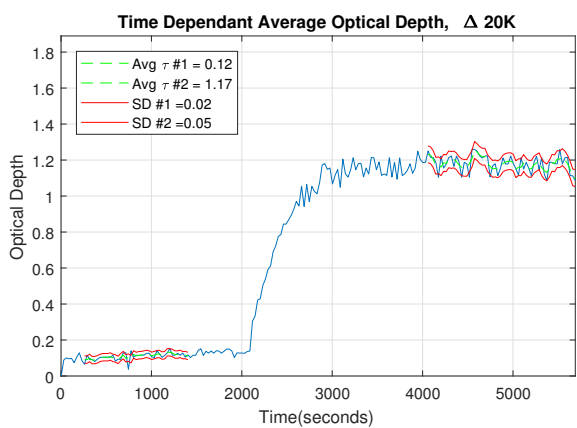

(d) Hygrometer Results at 20K/m Gradient

Figure 3.3: Laser - Hygrometer results showing the OD before and after the introduction of aerosols into the cloud chamber. The extinction caused by the water vapor, represented by the first mean line, is subtracted from the overall extinction after 90 minutes, represented by the second mean line. This results in the measured OD only being due to light scattering from water droplets. The dashed green line represents a running mean algorithm of 3 index values, which corresponds to a total of 90 seconds. The red lines represent one standard deviation from the mean, both below and above. Resulting in a capture of $\approx 68 \%$ of all data points taken from the LaserHygrometer.

consistent $\mathrm{OD}$ value of $\tau \approx 0.14$ before aerosols were injected, it indicates that the conditions inside the $\pi$ - Chamber were clean and ideal. Furthermore, small deviations between optical depth values before aerosols inputs show that the bulk turbulent conditions of supersaturated air plays a minimal role in altering the angle of arrival (AoA) of the laser beam as shown in similar example using water-tanks, Pawar and 
Arakeri, 2016),(Yuan et al., 2014),(Consortini et al., 1980). Otherwise the extinction

at higher temperature differences would be far larger and more varied as the beam jumps in and out of the optical sensor. For all cases, the extinction rate stabilized near the end of the 90 minute mark. This can be interpreted as the conditions inside reaching steady-state. As the gradient $(\mathrm{K} / \mathrm{m})$ increased so did the overall OD post aerosol-injection. This is due to larger gradients $(\mathrm{K} / \mathrm{m})$ creating a more supersaturated and turbulent environment where eddys were more numerous and with larger vorticities. In turn the mixing inside the $\pi$-Chamber was more pronounced which lead to the creation of activated water droplets, rather then inactivated hydrated aerosols.

\subsection{Hygrometer and WELAS Comparison}

\subsubsection{Results}

We compare the OD of the WELAS for droplets located at three different locations inside the $\pi$ - Chamber and the Laser-Hygrometer in figure (3.4). The comparison shows how alike and different the computed OD, via Mie Scattering algorithms, is from a direct transmission. Where the direct transmission comes from a real beam of light propagating through the cloud. 


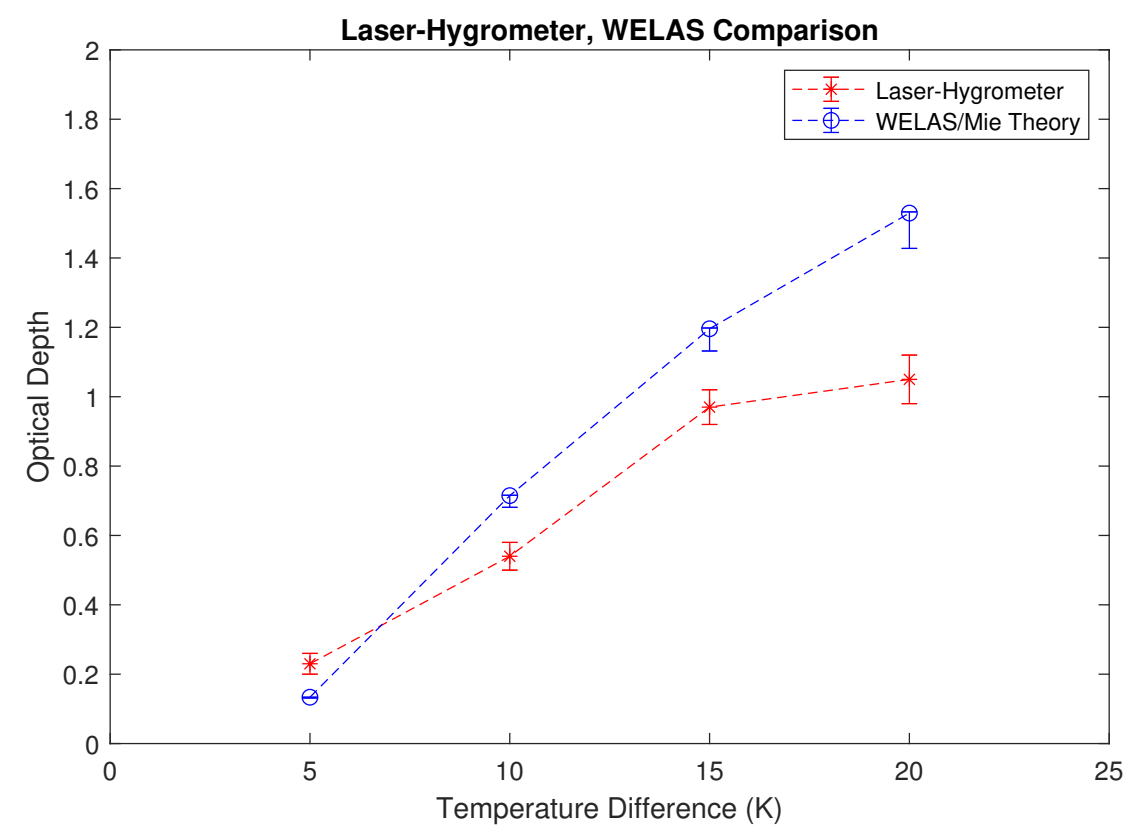

Figure 3.4: The OD results of both the WELAS and the Laser-Hygrometer. Temperature difference here represents the temperature between the floor and ceiling inside the $\pi$-Chamber. The error margin for the WELAS comes from the uncertainty for the location of the particles inside the $\pi$-Chamber with respect to the experimental photo-diode. The errors for the LaserHygrometer come from the oscillating nature of the transmission ratio during the experiment. This sources of the oscillation could be from experimental equipment such as $V_{\text {Tec }}, V_{\text {Bias }}$ signal not outputting correctly, and/or rapid temperature changes causing fluctuations of the lasers emitted wavelength. Another possible source of the fluctuation could be due to the particle clustering due to turbulence inside the $\pi$-Chamber. They represent one standard deviations above and below the mean OD value, capturing up to $68 \%$ of possible values.

\subsubsection{Analysis}

From the results of the WELAS and Laser-Hygrometer, we see a strong similarities in OD at smaller gradient $(\mathrm{K} / \mathrm{m})$ values, where the turbulence should be at its smallest and in turn the droplet distribution correlation is near homogeneity. But as the 
gradient $(\mathrm{K} / \mathrm{m})$ increases the two OD values diverge significantly. 


\section{Chapter 4}

\section{Conclusion}

\subsection{Overall Implications}

The comparison between the computational, and experimental results suggests that there could exists a divergence of light extinction from its expected exponential curve derived from Beer-Lambert's Law for a non-correlated random medium. The results agreed with our expected outcome, that a particle filled turbulent medium will likely increase the mean free path of light. This suggests that clouds could operate as a far more complex variable than previously expected. Our comparison showed a pseudo positively-correlated medium, meaning that light was able to propagate through the

cloud, while it's overall intensity diminished less than expected. When comparing our 
results to the simulation found in figure (1.1), the deviation of optical depth is larger then expected. Suggesting that other factors, such as multiple scattering, could be playing a role in lowering our OD from the Laser-Hygrometer further than the effects of turbulence. In a real world remote sensing scenario, this could translate into an object appearing closer to the instrument than it actually is.

\subsection{Limitations and Issues}

As stated previously, one of the biggest issues faced through the experiment was the presence of fog and droplets on windows which would cause issues for the optic readings from the Laser-Hygrometer and PDI. If conditions were found to be too moist within the $\pi$-Chamber, then the experimental photo-diode would be completely blocked when a small temperature difference was applied due to the resulting fog build up on the window. Because of this we have to operate under non-wetted side-wall conditions. We checked for foggy windows throughout the experiment to make sure conditions were optimal. If the window did become compromised with fog or water, it would be very clear in the data from the Laser-Hygrometer. A large and rapid spike in the OD that peaked at $\tau \approx 4$ would form and persist until the windows were cleaned, or conditions significantly changed. The condensation on the windows also made it difficult to obtain more than four final data points to analyze. An example of a failed experiment where the windows became too foggy can be found in the appendix. 
One of the most valuable features of the $\pi$-Chamber, the ability to create expansion clouds (Chang et al., 2016), was not used due to the limitations of the WELAS. Since the WELAS operated by sucking in air and performing optical analysis of particles as they passed through, we feared that the presence of a pressure difference could cause damage to the sensitive optics inside. Additionally this would create an ideal scenario for the PDI to be used, instead of the WELAS, to measure the DSD, but expansion cloud were also very troublesome due to it's tendency to create foggy windows. This in effect limited the research to steady-state, dry-wall, vertical temperature difference conditions so that both the WELAS and Laser-Hygrometer could ideally operate.

Another parameter that is difficult to properly characterize and quantify is the asymmetric parameter $\mathrm{G}$ for multiple scattering. The larger the parameter becomes the more occurrences of multiple scattering we could experience, if light is able to both scatter out of the path towards the photo-diode, and then scatter back in, at a non-trivial probability then it could explain the deviation between computed and experimental OD. Since computationally it would be registered as lost light, but experimentally it would still be viewed by the experimental photo-diode. While quantifying its overall effect can be difficult, reducing its influence could be another alternative by reducing the beam size and opening in which it could be detected. This would involve the focusing of the beam to a finer point, and the application of possible tubing that leads to the window, effectively reducing the angle window in which light could scatter multiple times and still be detected. And in turn reducing the influence that 
multiple scattering could play in the experiment.

\subsection{Future Research Possibilities}

This research topic is ripe with possibilities for future expansions. If the issue of the foggy windows can be fixed, we can introduce the PDI and expansion-clouds into the research. Many suggestions have already been placed in regards to how to solve this problem. One suggestion includes the placement of a small windshield wipers, set with a repeating timer, on the inside window that cleans the AoI while leaving minimal streaks. Another possibility is to heat the windows themselves, so that water does not condense on it. And lastly, a small air canister aimed towards the AoI that pushes away the condensation. This added features could allow us to take far more data points to get a clearer image of the properties of light propagation in a turbulent medium. Additionally, adding the Particle Image Interferometer (PIV) into this research could be beneficial. PIV is capable of interpreting the change in location for particles being lit by a plane laser into two-dimensional velocity components. The abilities of the PIV can help us quantify the vorticity rates inside the $\pi$-Chamber and allow us to get a better understanding of the flow parameters and turbulent mechanisms in play that may have caused the creation of a pseudo positively-correlated medium. 
The WELAS and the PDI are both effective in creating DSD of the conditions inside the $\pi$-Chamber. But they have different degrees of effectiveness at different sizes for droplets. Where the WELAS is better at small sizes below $10 \mu m$-radius sizes, while the PDI is more effective above the same radius size. This causes issues when trying to compare their DSD, when the mean droplet size is in the middle. With the window issue resolved, further research can be made into creating a combined DSD from the PDI and the WELAS where the Laser-Hygrometer can be used as a mediating tool in low turbulent environments where we are unsure of which instrument to trust more at each size bin. Analysis on expansion clouds can also be done with the use of the PDI when the foggy window issue is resolved.

In (Pawar and Arakeri, 2016), the frequency spectra was taken from a buoyancy driven turbulent environment. They found a unique, previously unknown of, scaling for Angle of Arrival(AoA) fluctuations in lower frequency. Where the fluctuations come from a beam propagating through a turbulent medium, and being deflected due to it. We believe that it would be interesting if their results could be replicated withing the $\pi$-Chamber under non-cloudy conditions. This would require more in-depth modifications of the Laser-Hygrometer, including but not limited to the replacement of the experimental photo-diode with a translucent plate and a camera, for recording, behind it. The code for AoA fluctuations tracking would also need to be developed. In addition to the replication of results found in (Pawar and Arakeri, 2016), the introduction of scattering particles such as droplets would be an interesting venture 
into possible unknown scaling parameters; if they exist in such environments.

With a better understanding of how light propagates in clouds, both steady-state and expansion, we can then start applying and relating conditions inside the $\pi$-Chamber to real world atmospheric scenarios. This could lead to a better understanding of how cloud cover should affect the signal strength of remote sensing instruments so that the data from these instruments does not result in over or underestimations, and further reduce the uncertainty found in radiation propagation through cloud cover. 


\section{References}

Bohren, C., and D. R. Huffman, Absorption and Scattering of Light by Small Particles, Wiley Science Paperback Series, 1998.

Borovoi, A., On the extinction of radiation by a homogeneous but spatially correlated random medium: comment, J. Opt. Soc. Am. A, 19(12), 2517-2520, doi:10.1364/ JOSAA.19.002517, 2002.

Buck, A. L., New equations for computing vapor pressure and enhancement factor, Journal of Applied Meteorology, 20(12), 1527-1532, doi:10.1175/1520-0450(1981) 020〈1527:NEFCVP $>2.0 . C O ; 2,1981$.

Chandrakar, K. K., W. Cantrell, K. Chang, D. Ciochetto, D. Niedermeier, M. Ovchinnikov, R. A. Shaw, and F. Yang, Aerosol indirect effect from turbulence-induced broadening of cloud-droplet size distributions, Proceedings of the National Academy of Sciences, 113(50), 14,243-14,248, doi:10.1073/pnas.1612686113, 2016.

Chang, K., et al., A laboratory facility to study gasaerosolcloud interactions in a 
turbulent environment: The chamber, Bulletin of the American Meteorological Society, 97(12), 2343-2358, doi:10.1175/BAMS-D-15-00203.1, 2016.

Consortini, A., P. Pandolfini, C. Romanelli, and R. Vanni, Turbulence investigation at small scale by angle-of-arrival fluctuations of a laser beam, Optica Acta: International Journal of Optics, 27(8), 1221-1228, doi:10.1080/713820367, 1980.

Davis, A. B., and M. B. Mineev-Weinstein, Radiation propagation in random media: From positive to negative correlations in high-frequency fluctuations, Journal of Quantitative Spectroscopy and Radiative Transfer, 112(4), 632-645, doi:10.1016/j. jqsrt.2010.10.001, 2011.

Du, H., Mie-scattering calculation, Appl. Opt., 43(9), 1951-1956, doi:10.1364/AO.43. 001951, 2004.

eblanaphotonics, DX1 Laser Diode Controller Module, eblanaphotonics.

Frankel, A., G. Iaccarino, and A. Mani, Convergence of the bouguerbeer law for radiation extinction in particulate media, Journal of Quantitative Spectroscopy and Radiative Transfer, 182, 45 - 54, doi:https://doi.org/10.1016/j.jqsrt.2016.05.009, 2016.

Frankel, A., G. Iaccarino, and A. Mani, Optical depth in particle-laden turbulent flows, Journal of Quantitative Spectroscopy and Radiative Transfer, 201, 10 - 16, doi:https://doi.org/10.1016/j.jqsrt.2017.06.029, 2017. 
Gogoi, A., A. Choudhury, and G. A. Ahmed, Mie scattering computation of spherical particles with very large size parameters using an improved program with variable speed and accuracy, Journal of Modern Optics, 57(21), 2192-2202, doi:10.1080/ 09500340.2010.533206, 2010.

Gordon, I., et al., The hitran2016 molecular spectroscopic database, Journal of Quantitative Spectroscopy and Radiative Transfer, 203, 3 - 69, doi:https://doi.org/10. 1016/j.jqsrt.2017.06.038, hITRAN2016 Special Issue, 2017.

Hale, G. M., and M. R. Querry, Optical constants of water in the 200-nm to $200-\mu \mathrm{m}$ wavelength region, Appl. Opt., 12(3), 555-563, doi:10.1364/AO.12.000555, 1973.

Kostinski, A. B., On the extinction of radiation by a homogeneous but spatially correlated random medium, J. Opt. Soc. Am. A, 18(8), 1929-1933, doi:10.1364/ JOSAA.18.001929, 2001.

Kostinski, A. B., On the extinction of radiation by a homogeneous but spatially correlated random medium: reply to comment, J. Opt. Soc. Am. A, 19(12), 25212525, doi:10.1364/JOSAA.19.002521, 2002.

Larsen, E. W., and R. Vasques, A generalized linear boltzmann equation for nonclassical particle transport, Journal of Quantitative Spectroscopy and Radiative Transfer, 112(4), 619 - 631, doi:https://doi.org/10.1016/j.jqsrt.2010.07.003, 2009 International Conference on Mathematics and Computational Methods (MC 2009), 2011. 
Larsen, M. L., and A. S. Clark, On the link between particle size and deviations from the beerlambertbouguer law for direct transmission, Journal of Quantitative Spectroscopy and Radiative Transfer, 133, 646 - 651, doi:https://doi.org/10.1016/ j.jqsrt.2013.10.001, 2014.

Matzler, C., Matlab functions for mie scattering and absorption, IAP Res Rep, 8, 2002.

NI, NI PCI-6110/6111 Specifications, National Instruments, 2004.

Packard, C. D., R. A. Shaw, W. H. Cantrell, G. M. Kinney, M. C. Roggemann, and J. R. Valenzuela, Measuring the detector-observed impact of optical blurring due to aerosols in a laboratory cloud chamber, Journal of Applied Remote Sensing, 12(4), 1 - 21 - 21, doi:10.1117/1.JRS.12.042404, 2018.

PALAS, OPERATING MANUAL WELAS DIGITAL2000 SERIES, PALAS.

Pawar, S. S., and J. H. Arakeri, Intensity and angle-of-arrival spectra of laser light propagating through axially homogeneous buoyancy-driven turbulence, Appl. Opt., 55(22), 5945-5952, doi:10.1364/AO.55.005945, 2016.

Petty, G., A first course in atmospheric radiation, Sundog Publishing, 1998.

Shah, G. A., Numerical methods for Mie theory of scattering by a sphere., Kodaikanal Observatory Bulletins, 2, 42-63, 1977. 
Shaw, R. A., A. B. Kostinski, and D. D. Lanterman, Super-exponential extinction of radiation in a negatively correlated random medium, Journal of Quantitative Spectroscopy and Radiative Transfer, 75(1), 13 - 20, doi:https://doi.org/10.1016/ S0022-4073(01)00287-4, 2002.

Stephens, G., D. Winker, J. Pelon, C. Trepte, D. Vane, C. Yuhas, T. LEcuyer, and M. Lebsock, Cloudsat and calipso within the a-train: Ten years of actively observing the earth system, Bulletin of the American Meteorological Society, 99(3), 569-581, doi:10.1175/BAMS-D-16-0324.1, 2018.

Thorlabs, SM05PD6A - Large Area Mounted Germanium Photodiode, 800-1800 nm, Cathode Grounded, Thorlabs.

Twomey, S., Pollution and the planetary albedo, Atmospheric Environment (1967), 8(12), 1251 - 1256, doi:https://doi.org/10.1016/0004-6981(74)90004-3, 1974.

Twomey, S., The influence of pollution on the shortwave albedo of clouds, Journal of the Atmospheric Sciences, 34(7), 1149-1152, doi:10.1175/1520-0469(1977) 034〈1149:TIOPOT $\rangle 2.0 . \mathrm{CO} ; 2,1977$.

Wang, R. T., and H. C. van de Hulst, Rainbows: Mie computations and the airy approximation, Appl. Opt., 30(1), 106-117, doi:10.1364/AO.30.000106, 1991.

Wiscombe, W. J., Mie scattering calculations: Advances in technique and fast, vectorspeed computer codes, NCAR Technical Notes, doi:10.5065/D6ZP4414., 1979. 
Yuan, R., J. Sun, T. Luo, X. Wu, C. Wang, and C. Lu, Simulation study on light propagation in an isotropic turbulence field of the mixed layer, Opt. Express, 22(6), 7194-7209, doi:10.1364/OE.22.007194, 2014. 
Appendix A

Other Plots and Images 

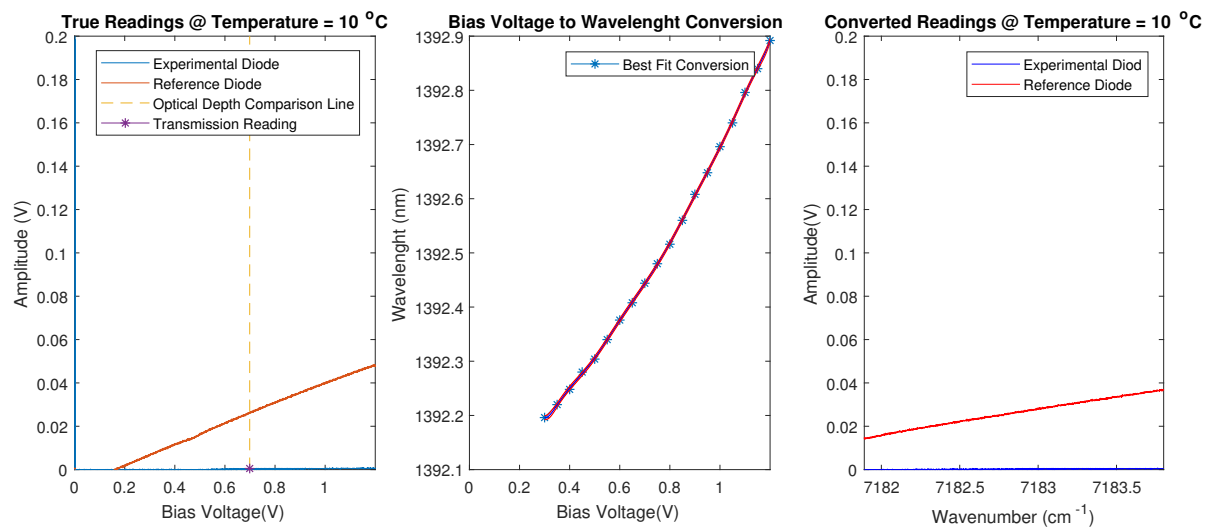

(a) Laser-Hygrometer Data

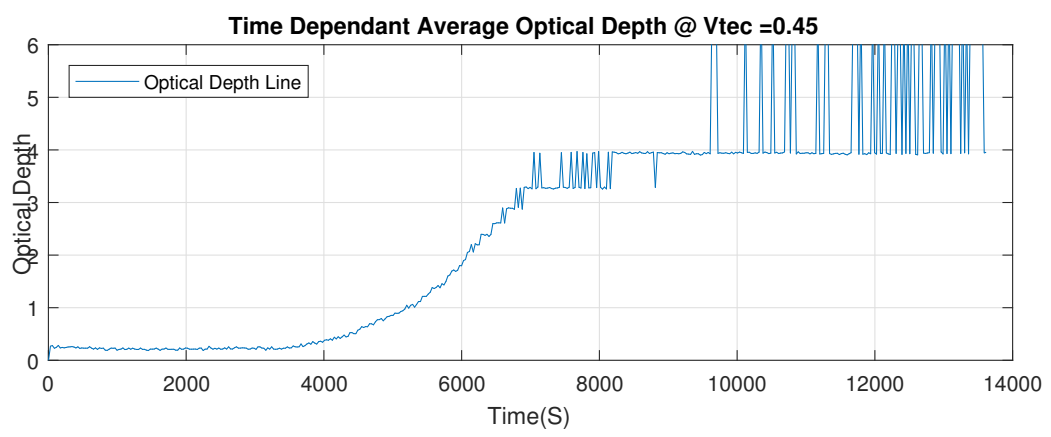

(b) Optical Depth Line

Figure A.1: This is the result obtained when the windows become foggy. A maximum optical depth of $\approx 4$ is reached. When that occurs the experimental photo-diode is flattened out and the calculations of optical depth become infinity due to a division by zero. 


\section{Appendix B}

\section{Letters of Permission}

\section{B.1 Permission From AMS}

Hello Eduardo,

Thank you for the additional information. This signed message constitutes permission to use the material requested below.

You may use the figure in your upcoming publication with the following conditions:

1. Include the complete bibliographic citation of the original source. 2. Include the following statement with that citation: American Meteorological Society. Used with 
permission.

If you have any questions or need additional information, please feel free to contact me.

*Please note: If the material in an AMS journal is credited to another source, the requester must obtain permission or license from that source directly. That material may not be used without permission or license from the copyright holder.*

Best,

\section{B.2 Elsevier}

This Agreement between Michigan Technological University - Eduardo Rodriguez-feo ("You") and Elsevier ("Elsevier") consists of your license details and the terms and conditions provided by Elsevier and Copyright Clearance Center.

License Number - 4564290149954

License date - Apr 08, 2019

Licensed Content Publisher - Elsevier 
Licensed Content Publication - Journal of Quantitative Spectroscopy and Radiative Transfer

Licensed Content Title - Super-exponential extinction of radiation in a negatively correlated random medium

Licensed Content Author - Raymond A Shaw,Alexander B Kostinski,Daniel D Lanterman

Licensed Content Date - Aug 1, 2002

Licensed Content Volume - 75

Licensed Content Issue - 1

Licensed Content Pages - 8

Start Page - 13

End Page - 20

Type of Use - reuse in a thesis/dissertation

Portion - figures/tables/illustrations

Number of figures/tables/illustrations - 1 
Format - both print and electronic

Are you the author of this Elsevier article? - No

Will you be translating? - No

Original figure numbers - Instead of using figures 1, and 2 I decided to use figure 3 as it better relates to my research.

Title of your thesis/dissertation - LIGHT PROPAGATION THROUGH A TURBULENT MEDIUM

Expected completion date - May 2019

Estimated size (number of pages) - 40

Requestor Location -

Michigan Technological University 1107 E Houghton Ave

HOUGHTON, MI 49931 United States Attn: Michigan Technological University

Publisher Tax ID - 98-0397604

Total - 0.00 USD 